\title{
Modelling the nonlinear behaviour of an underplatform damper test rig for turbine applications
}

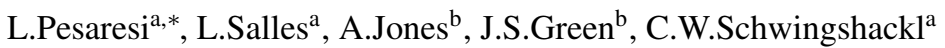 \\ ${ }^{a}$ Imperial College London, London SW7 2AZ, UK \\ ${ }^{b}$ Rolls-Royce plc, Derby DE2 48J, UK
}

\begin{abstract}
Underplatform dampers (UPD) are commonly used in aircraft engines to mitigate the risk of high-cycle fatigue failure of turbine blades. The energy dissipated at the friction contact interface of the damper reduces the vibration amplitude significantly, and the couplings of the blades can also lead to significant shifts of the resonance frequencies of the bladed disk. The highly nonlinear behaviour of bladed disks constrained by UPDs requires an advanced modelling approach to ensure that the correct damper geometry is selected during the design of the turbine, and that no unexpected resonance frequencies and amplitudes will occur in operation. Approaches based on an explicit model of the damper in combination with multi-harmonic balance solvers have emerged as a promising way to predict the nonlinear behaviour of UPDs correctly, however rigorous experimental validations are required before approaches of this type can be used with confidence.

In this study, a nonlinear analysis based on an updated explicit damper model having different levels of detail is performed, and the results are evaluated against a newly-developed UPD test rig. Detailed linear finite element models are used as input for the nonlinear analysis, allowing the inclusion of damper flexibility and inertia effects. The nonlinear friction interface between the blades and the damper is described with a dense grid of 3D friction contact elements which allow accurate capturing of the underlying nonlinear mechanism that drives the global nonlinear behaviour. The introduced explicit damper model showed a great dependence on the correct contact pressure distribution. The use of an accurate, measurement based, distribution, better matched the nonlinear dynamic behaviour of the test rig. Good agreement with the measured frequency response data could only be reached when the zero harmonic term (constant term) was included in the multi-harmonic expansion of the nonlinear problem, highlighting its importance when the contact interface experiences large normal load variation. The resulting numerical damper kinematics with strong translational and rotational motion, and the global blades frequency response were fully validated experimentally, showing the accuracy of the suggested high detailed explicit UPD modelling approach.
\end{abstract}

Keywords: Damper model, friction damping, turbine blade vibrations, nonlinear dynamics, bladed disks, passive control

\section{Introduction}

Continuous demand in the aerospace industries for lighter and more efficient gas turbine engines has driven the design of many components to their structural limits. This applies to the case of turbine blades, which are highly loaded components, carrying high thermal and centrifugal stresses as well as stresses caused by the blade vibrations [1]. These latter are high frequency alternating resonance stresses, and can lead to high cycle fatigue (HCF) [2] and eventual failure of the blades. However, the wide operating speed range of aero-engines together with the high modal density of bladed disks makes it impossible to avoid all critical resonances during operation. Reducing the vibration

\footnotetext{
*Corresponding author.

E-mail address : luca.pesaresi12@imperial.ac.uk.
}

Tel.: +44 (0)207 5942117 
amplitude at those resonances is therefore crucial, and passive systems, based on friction damping [3, 4], have been the most widely used approach over the years [5].

Dry friction can provide damping in various locations on a blade, such as the shrouds, roots and blade tips, and the most effective way to exploit it relies on underplatform dampers (UPD) [5]. Underplatform dampers consist of a metal device which sits in a groove on the underside of the platform between adjacent blades, and it is kept in place and loaded by the centrifugal force. When the blades vibrate, the relative motion between the adjacent platforms and the damper leads to friction at the contact interface, which in turn provides energy dissipation and damping to the system [6, 7]. The dynamics of a bladed disk assembly constrained by UPDs is governed by nonlinear differential equations, due to the nonlinear nature of the forces occurring at the interface [1]. The main sources of this nonlinearity are the localised transition from stick to slip and a unilateral normal force which can lead to gaps at the contact interface.

Many studies on damper modelling have been conducted [8-12], all leading to a better understanding of the damper behaviour, but despite all the research efforts, there is still not a well established approach available today. Initially [8, 13], the blade damper system was reduced to a single degree of freedom oscillator with a spring element combined to a Coulomb friction element (Jenkins element [3]) and a semi-analytical solution was derived. In [14] an attempt to improve this model was carried out, by extending it to a two-degrees of freedom system. A higher fidelity was achieved by full FE models of the blades in conjunction with kinematic hypotheses about the relative motion between the damper and platform, in which the damper and platform faces are always parallel [6, 9, 10, 15]. Difficulties encountered during the validation of the latter models suggested that the required hypotheses were not always realistic [6]. In more advanced models [16-20], these hypotheses were removed and the damper motion is calculated purely as the result of its interaction with the blades by means of the nonlinear contact forces. To reproduce damper motions, advanced 3D friction contact elements were employed; these account for normal load variation with separation effects and 2D tangential in plane motion [21]. To further improve the accuracy of the contact force calculation, local deformation effects were also taken into account using an explicit FE damper model [22]. These improvements have led to the emergence of tools that permit an accurate representation of the contact interface, leading to a potentially very large number of nonlinear equations to solve.

A possible approach to solving the nonlinear equations of motion of the dynamic systems is time integration, but it is computationally expensive and highly inefficient when the interest is only the steady-state response to harmonic excitation. For this reason, a combination of the harmonic balance method (HBM) [18, 23, 25] which is based on a fundamental harmonic or multi-harmonic approximation of the nonlinear forces, together with model reduction techniques [26-28] has emerged as a widely used approach to solve vibration problems.

Significant advances in the damper models over the last years have not always been accompanied by the rigorous validation process needed for reliable predictions. Higher fidelity models [16, 19, 22] comprise several parameters describing the contact interface but their impact in capturing the physics of the phenomena needs to be fully understood before those models can be used with confidence. The aim of the present work is to improve the fidelity of the current explicit damper modelling approach [22], to better capture the strong nonlinear dynamics often observed in real environments [29]. A dedicated UPD test rig was designed for this scope, based on a double beam configuration [6, 11, 30, 32]. Unlike similar set-ups tested, particular care was taken to define a geometry able to mimic the dynamics of a real high-pressure turbine (HPT) blade. A high fidelity 3D nonlinear model was created and its results are compared to the measured data. Detailed model updating and a parameter study of the main model parameters led to a more accurate understanding of the nonlinear mechanisms at the contact interface and a significantly improved representation of the highly nonlinear response of the system.

\section{UPD rig design}

The validation of a nonlinear dynamic analysis of UPDs requires reliable test data from a well-controlled environment to ensure that all the required parameters can be identified and incorporated in the analysis. A dedicated UPD test rig was developed and used for the validation in the subsequent analysis.

\subsection{Rig concept and Non-dimensional parameters}

The underplatform damper test rig developed for this study is an experimental set up that allows the effect of UPDs on blade-like structures to be evaluated [33]. Following a review of similar test rigs [6, 11, 31, 32], a static 
rig design was chosen in order to minimise the effect of mistuning on the response, as well as allow better control of the experimental set up, and simplify the testing procedure. In recent developments [34, 35], a specific test rig was designed to measure the forces transmitted between the damper and the platform, as well as the relative displacements, allowing a fine tuning of the contact parameters when compared to the simulations. More realistic set ups have been proposed [36, 37], but they tend to introduce additional complications such as uncertainties about the damper position during operation, and mistuning due to the bladed disk manufacturing tolerances. The asymmetry of the excitation system used in the double beam configuration could lead to potential mistuning as well, but with the damper in place, no double peak response was observed for the analysed modes.

The basic concept of the rig can be seen in Fig 1a. Two pseudo beam-like blades are fixed on a common base, which simulates a rigid disk. For this investigation, the damper is a wedge-type, [36], which has a triangular cross section with a characteristic angle. Unlike in a real high-pressure turbine blade, the aerofoil is substituted by a straight rectangular cross-section beam. To ensure that these simplifications do not lead to non-representative nonlinear dynamic behaviour, the geometry of the pseudo blades has been designed to mimic the dynamic response of a real HPT blade. For this purpose, a set of non-dimensional design parameters has been introduced (see Table $1 \mathrm{~b}$ ), characterising the main aspects of the dynamic behaviour of a turbine blade. These parameters, in part selected from the literature [6, 13, 38] and in part newly defined, are:

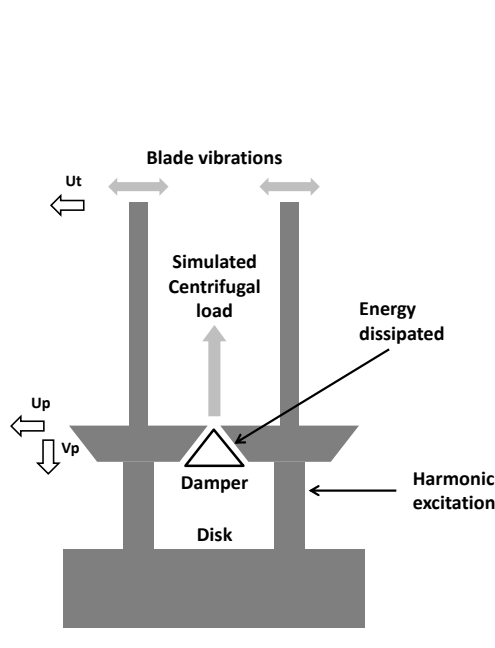

(a)

\begin{tabular}{lcc}
\hline & Description & Expression \\
\hline A) & Force ratio & $\frac{C F}{F_{e x c}}$ \\
B) & Acceleration ratio & $\frac{r \Omega^{2}}{a \omega^{2}}$ \\
C) & Displacement ratio & $\frac{u_{p}}{u_{t}}$ \\
D) & Platform displacement ratio & $\frac{u_{p}}{v_{p}}$ \\
E) & Frequency shift & $\frac{\omega_{d}}{\omega_{u}}$ \\
F) & Energy ratio & $\frac{E_{f r i}}{E_{t o t}}$ \\
G) & Contact strain & $\frac{\sigma}{E}$ \\
\hline
\end{tabular}

(b)

Figure 1: a) Basic concept of the UPD rig, b) table of non-dimensional parameters used to define similar dynamic behaviour between the rig and real HPT blades.

A) the force ratio [13] of the centrifugal load over the excitation force, which controls the regime in which the damper is operating. At a low ratio, the excitation force is predominant and the damper is in slip condition, whereas at very high levels the damper is in full stuck condition. During the measurement this parameter can be varied by modifying the damper load or the shaker force.

B) the acceleration ratio, which in a real engine, represents the ratio between the centrifugal acceleration of the damper, due to the rotational speed, and the acceleration due to the vibration of the blade platform projected along the same direction. This parameter controls whether or not the damper separates from the platform when the damper acceleration is exceeded by the platform acceleration. In the simplified test rig the damper maximum acceleration is governed by the flexibility of the wire-pulley system. 
C) the displacement ratio [38], which is the ratio between the horizontal displacement of the platform and the horizontal displacement of the blade tip in the first flexural mode of the blade. Higher values cause more relative motion at the damper interface, leading to more energy dissipated. It allows similar mode shapes between the real blade and the test rig.

D) the platform displacement ratio [6], which is the ratio between the horizontal and vertical displacement of the platform. This parameter influences the kinematics of the damper during a vibration cycle and ensures realistic damper motions.

E) the frequency shift, which indicates the change in frequency caused by the damper compared to the baseline configuration (no damper). The damper causes the stiffening of the system due to the coupling of the blades, which depends on the load, on the mode considered, and on the platform and damper geometry. It will ensure that the chosen design leads to realistic frequency shifts.

F) the energy ratio, which considers the energy dissipated by the friction damping and the energy dissipated due to the material damping. It quantifies the contribution of the damper to the total energy dissipation in the dynamic system.

G) the contact strain, which is the ratio between the contact pressure and the Young's modulus of the material used at the interface. Keeping it similar to a real blade allows the rig to reproduce a similar pressure distribution and similar wear behaviour.

\subsection{Design features and set-up}

The design process of the UPD test rig was driven by the above mentioned parameters and by the need of minimising external factors that could affect the quality of the nonlinear measurements. The geometry of the blades was defined and optimised using linear FE and nonlinear dynamic analysis, leading to the final design shown in Fig. 3a. When evaluating the nonlinear dynamic response caused by friction damping, it is very important that the underlying dynamic system without a damper is linear, and the nonlinearities introduced by possible external sources are minimal [39. 41]. For this reason the fir-tree attachment that would connect the real blade to the disk was removed here to minimise the number of friction joints, and the blades were clamped tightly to a two ton inertia block via a hydraulic vice capable of $200 \mathrm{kN}$ clamping force. Frequency sweeps of the rig without the damper at increasing clamping forces were performed, and the frequency and damping behaviour was extracted (Fig. 2) to gain an understanding of the performance of the clamp.

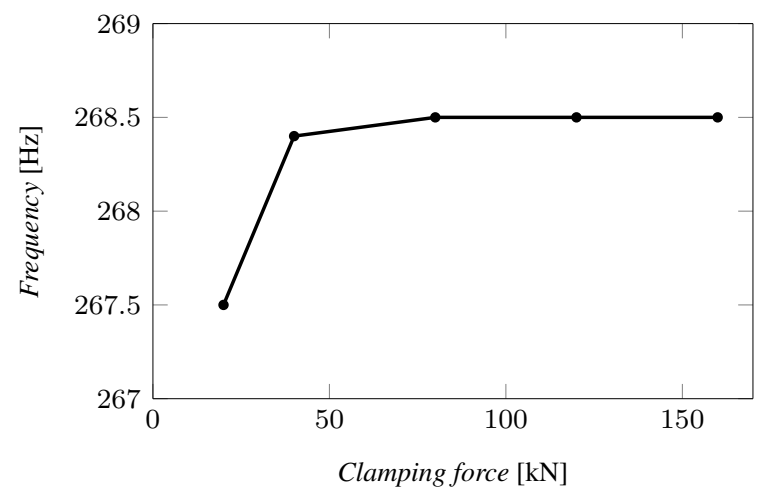

(a)

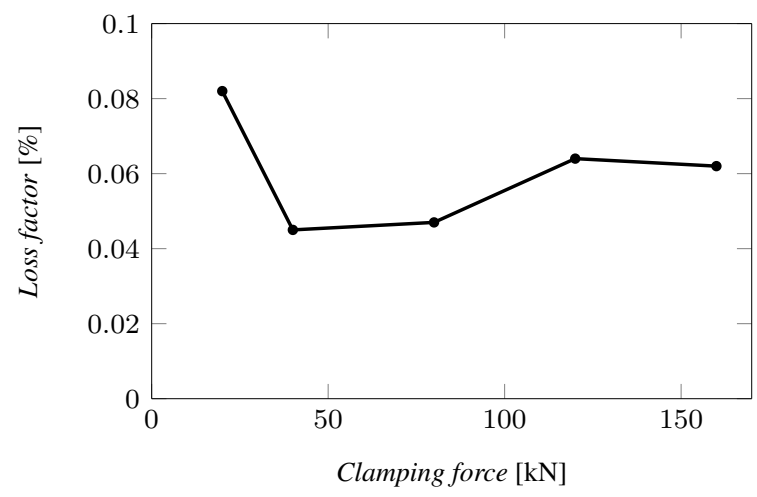

(b)

Figure 2: Clamping effect on the $1 \mathrm{~F}$ modal parameters a) Resonance frequency b) Loss factor.

Both loss factor and resonance frequency show some small variations at low clamping forces but seem to converge above $30 \mathrm{kN}$. The small influence of the clamp on the dynamics of the blades is further confirmed by the values of the loss factor which are below $0.1 \%$, which was very close to the free-free configuration of the blade. 
The centrifugal force, which the damper experiences in a real engine, is simulated with a pulley system and a set of variable weights. Since it was not possible to replicate the same acceleration ratio (section 2.1) experienced in a real engine (which is extremely high due to the high centrifugal load), a minimum condition was defined to avoid a complete separation at the damper-platform interface. This was achieved by selecting the stiffness $K_{w}$ of the stainless steel pulling wire to fulfil Eq, 1, as this guarantees that the restoring forces of the wire are higher than the inertia forces acting on the damper:

$$
K_{w}=\frac{E_{w} A}{l}>m \omega^{2}
$$

where $K_{w}$ is the stiffness of the wire, $E_{w}$ is the equivalent Young's modulus of the wire, $A$ is the nominal cross section area of the wire, $l$ is the free length between the damper and the first pulley, $m$ is the mass of the damper and $\omega$ is the vibration frequency of the blades.

The measurement set up can be seen in Fig. $3 b$. The excitation is provided by an electrodynamic shaker, attached below the platform, and the response is measured with two non-contact laser doppler vibrometers (LDV) focused 20 $\mathrm{mm}$ below the tip of each blade. A more detailed discussion of the rig development, and an initial assessment of its capabilities can be found in [33].

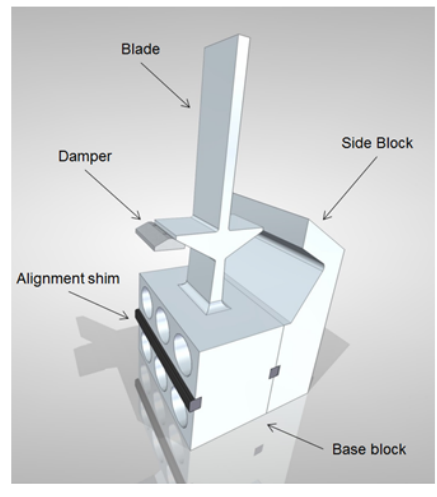

(a)

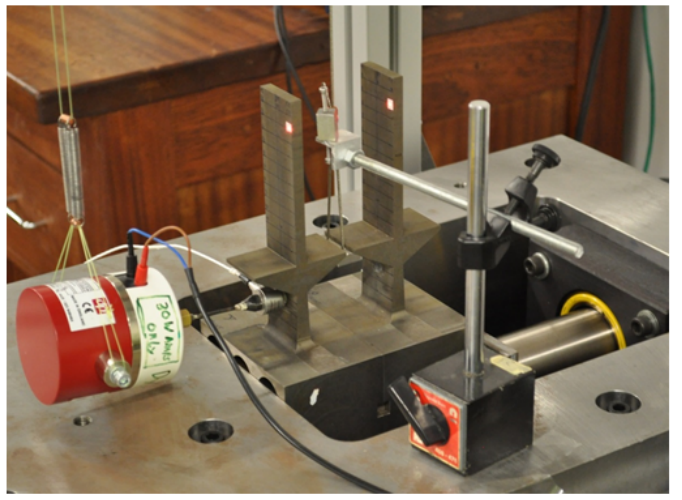

(b)

Figure 3: a) UPD rig final CAD design, single blade shown, b) UPD rig testing configuration: shaker excitation, blades hydraulic clamp and damper pulling system.

\section{Modelling approach}

\subsection{Method}

The approach used for the numerical analysis, is based on an exisiting multi-harmonic balance (MHBM) solver coupled with a model reduction technique, included in the code FORSE (FOrced Response SuitE) and discussed in detail in [21, 22, 28]. This flexible methodology can be applied to predict the nonlinear dynamic response of large FE models, which contain nonlinear joints and are subject to harmonic excitation. Due to the friction forces arising at the contact interface, the equations of motion for a blade-damper system are nonlinear, and can be written in the following form:

$$
\mathbf{M} \ddot{\mathbf{x}}(t)+\mathbf{C} \dot{\mathbf{x}}(t)+\mathbf{K} \mathbf{x}(t)+\mathbf{F}_{n l}(\dot{\mathbf{x}}(t), \mathbf{x}(t))=\mathbf{P}(t) .
$$

where $\mathbf{M}, \mathbf{C}, \mathbf{K}$ are the mass, damping, and stiffness matrices. $\mathbf{P}$ are the external excitation forces and $\mathbf{F}_{n l}$ are the nonlinear contact forces dependent on the relative motion of the interacting nodes at the interface. According to the 
MHBM, the response for each DOF of the system can be expressed as a Fourier series truncated at the $n^{\text {th }}$ harmonic:

$$
\mathbf{q}(\mathbf{t})=\mathbf{Q}_{0}+\sum_{j=1}^{n} \mathbf{Q}_{j}^{c} \cos m_{j} \omega t+\mathbf{Q}_{j}^{s} \sin m_{j} \omega t
$$

where $\mathbf{Q}_{0}, \mathbf{Q}_{j}^{c}$ and $\mathbf{Q}_{j}^{s}$ are the harmonic coefficients for each DOFs. When equation 3 is inserted in equation 2. and the harmonic terms balanced with a Galerkin projection, a system of equations to determine all harmonic components can be obtained in the frequency domain as follows:

$$
\mathbf{Z}(\omega) \mathbf{Q}+\mathbf{F}(\mathbf{Q})-\mathbf{P}=0
$$

where $\mathbf{Q}=\left\{\mathbf{Q}_{0}, \mathbf{Q}_{1}^{c}, \mathbf{Q}_{1}^{s}, \ldots, \mathbf{Q}_{n}^{c}, \mathbf{Q}_{n}^{s}\right\}^{T}$ is a vector of harmonic coefficients, $\mathbf{F}(\mathbf{Q})$ is the vector of nonlinear forces and $\mathbf{P}$ is the external excitation. $\mathbf{Z}(\omega)$ is the dynamic stiffness matrix of the system. To reduce the size of the problem, a model reduction based on a FRF matrix representation is applied, and the harmonic coefficients are calculated with a Newton-Raphson iterative solver. The FRF matrix is obtained by calculating its exact value at a particular frequency point, and including a second term which describes its variation in a wide range of frequency [28]. Thanks to this approach the accuracy of the contact displacement calculation is significantly improved, without the need to include too many modes in the truncated modal expansion.

\subsection{Steps of the analysis}

The steps required for the analysis are summarised in Fig. 4. Detailed FE models of both blades (54972 elements) and damper (3620 elements) were generated with quadratic hexahedral elements, and the material properties of the stainless steel used $(\mathrm{E}=197 \mathrm{GPa}$ rho $=7800 \mathrm{~kg} / \mathrm{m} 3)$ were used. The modal properties for the blades and the damper required for the model reduction were then extracted (24 modes) using SC03, a Rolls-Royce proprietary FE software.

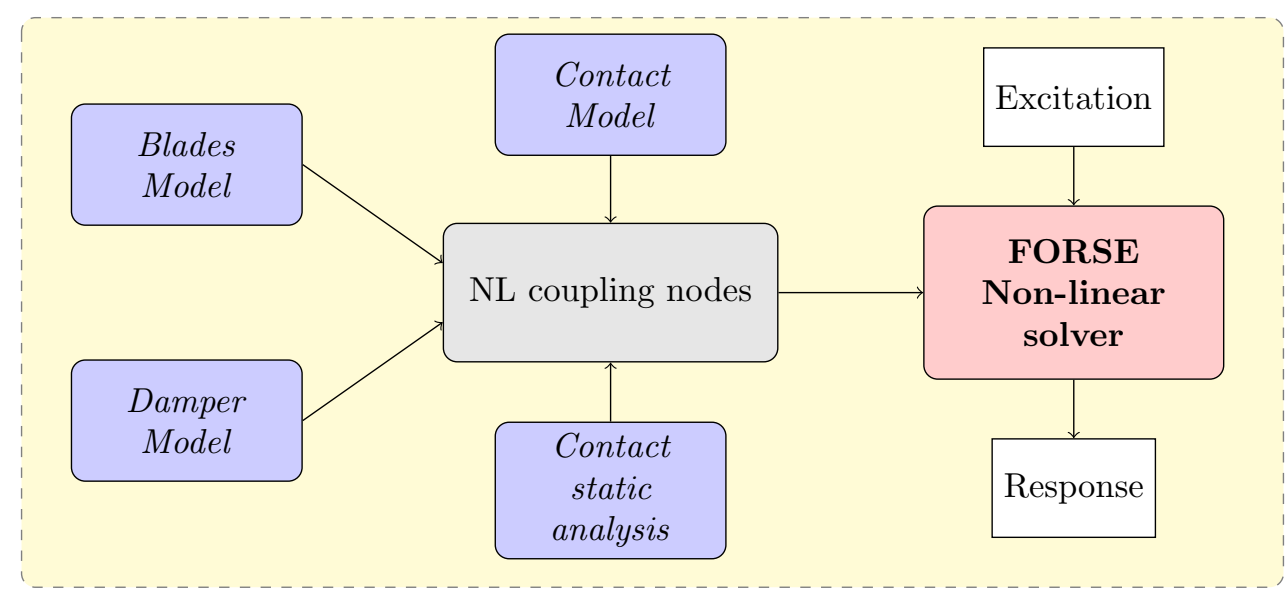

Figure 4: Steps required for the nonlinear simulation of the UPD rig.

The reduced blades and damper models are coupled by a set of contact nodes at the interface as shown in Fig.6a. Particular care was taken during the meshing process to guarantee matching nodes between the platform and the damper. To reduce the computational cost, only a subset of the FE interface contact nodes were selected. A coarser triangular mesh was superimposed to the contact FE mesh and only the FE mesh nodes closest to the centroids of the new triangles were selected. A total of 146 nodes were selected enabling a good discretization of the contact area, while keeping an acceptable computational time. The dynamic contact behaviour at each of these nodes is described by the 3D friction contact model shown in Fig. 6b This contact model comprise two decoupled Jenkins elements [3] allowing for 2D in plane motion, and a third spring element allowing for normal load variation. In addition, for each element, four parameters describing the contact properties need to be chosen: the friction coefficient $\mu$, the initial 
pre-load $\sigma_{0}$, and the tangential $K_{t}$ and normal $K_{n}$ stiffness. The stiffness values and the pre-loads are normalised by unit of area, so that each contact element has its specific stiffness and pre-load depending on the area of the triangular patch associated with it.

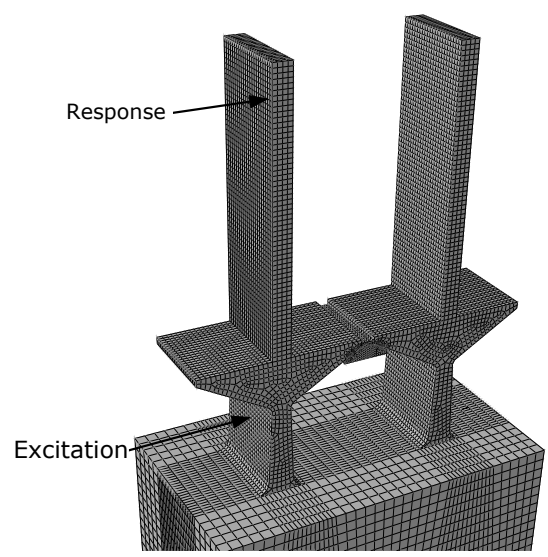

Figure 5: Blades and damper FE models.

To ensure an accurate starting point for the analysis, the linear model was validated, performing hammer tests on the blades without damper, and updating the material properties (Young's modulus and damping). Once the modal properties of the reduced linear model and the nonlinear element definition is available, the nonlinear analysis can be conducted. A single harmonic excitation, simulating the shaker, was applied to the closest node corresponding to the experimental position, and similarly, the response was evaluated near the blade tip as shown in Fig. 6b at the location of the LDVs. The first three harmonics, plus the harmonic "zero" (static term), were included in the Fourier truncated representation of the response, as an initial analysis showed that these were enough to capture the nonlinear phenomena observed (see section 5.3 .

A second, more simplistic approach was used to model the UPD rig dynamic response, as it allows very fast calculations, which are often useful during the preliminary design phase of an aero engine. With this approach, the damper is modelled as a lumped mass in its COG, and the interaction with the blades is described by a single contact element at the centroid of each damper face. Therefore, the localised nonlinear effect of stick-slip and separation are concentrated in one single point per interface and the damper flexibility is neglected. The damper motion is described by $\mathrm{X}, \mathrm{Y}$ and $\mathrm{Z}$ translation but no rotational DOF is included. This model implies a simplification of the damper motion and of the stick-slip phenomena, and represents a useful comparison to evaluate the contribution of the explicit damper model to capture the blade dynamic response. The results of the lumped mass damper simulations and a comparison to the explicit model will be discussed in section 6.1

\subsection{Input parameters study}

When modelling a damper, not much information is normally available about the properties of the contact, and some reference values for the describing parameters are chosen based on assumptions and experience. Normally, the static load on the damper due to the centrifugal force is assumed to be constant and equally distributed between the two platforms [6, 20]. However, when enough contact elements are used, non-uniform pressure distributions can be represented with a good approximation. To better understand the implications of the uniform pressure distribution assumption on the global dynamic response, an initial study was conducted with uniform and variable, and symmetric and asymmetric contact pressure distributions at the interface as described in section 5.1 .

Other important parameters required for the contact model are the friction coefficient $\mu$, and the tangential $K_{t}$ and normal $K_{n}$ stiffness. For the present analysis these parameters were measured experimentally with the 1D friction rig described in [42]. The friction coefficient can be measured with good confidence and an average value of $\mu=0.6$ was used for the analysis, but a much larger scatter on the tangential contact stiffness is normally experienced during the experiments. In addition, both contact normal and tangential stiffness change with normal load, however in the 


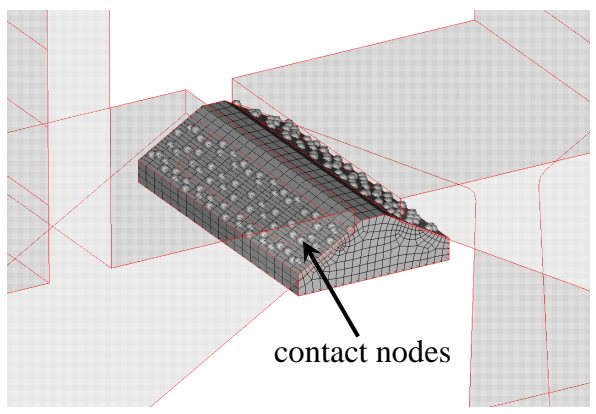

(a)

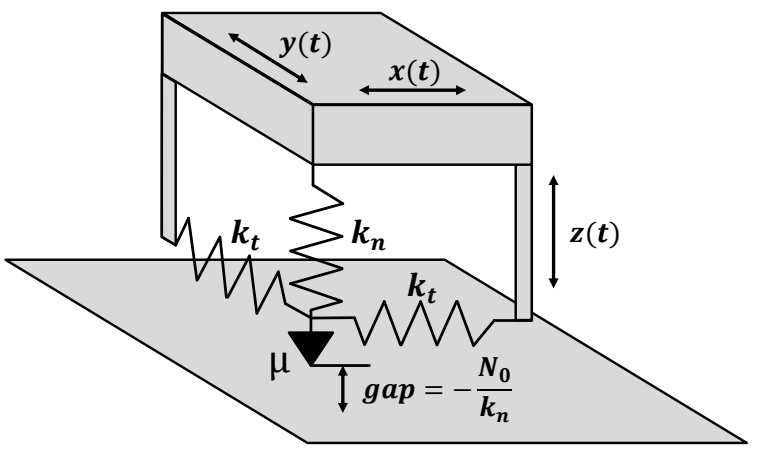

(b)

Figure 6: a) Contact nodes which couple the blade platforms and the damper at the interface, b) scheme of the 3D friction contact model employed.

contact model employed, only a constant value can be used. To evaluate the impact of a change in contact stiffness on the blade dynamics, different simulations were run with a range of $K_{t}$ and $K_{n}$ as described in section 5.2 .

In the current modelling approach, the static problem and the dynamic one are decoupled to allow any level of detail for the static analysis as discussed in section 6 . This means that the static load at the damper interface caused by the centrifugal force are calculated separately and not within the dynamic model. When this is the case, the "zero" harmonic term (static term) on the expansion of the NL forces is often neglected in the dynamic model. The impact of the inclusion of this term for the dynamic analysis of blade-damper models is shown in section 5.3 A previous study [18] addressed the importance of the "zero" harmonic term for the dynamic analysis, but the main difference with the present study is that a coupled static/dynamic analysis was used there.

\section{Nonlinear experimental FRFs and damper kinematics}

The nonlinear measurements were focussed on the first flexural mode (1F) where large platform movements were expected. Due to the double blade configuration of the rig with a single damper, two main 1F modes, an in phase (IP) and out of phase (OOP) can be excited. In a real engine, the inter blade phase angle (phase between two consecutive blades) will be between these two extreme values depending on the nodal diameter excited, so that the test rig covers the boundaries of the operational range of the damper. The damper was always loaded with a constant high load of $960 \mathrm{~N}$ to ensure a good conformity at the contact interface, since an initial convergence study showed strong joint variability at lower damper loads [33]. A stepped sine test with a Data Physics SignalCalc ACE card was performed around each resonance at various levels of excitation, from $0.01 \mathrm{~N}$ to $17 \mathrm{~N}$, leading to a $C F / F_{\text {exc }}$ ratio from $10 \mathrm{k}$ to 56. In order to have a level of control over the activation of the nonlinearity, a feedback control kept the excitation force constant throughout the sweep since amplitude control proved to be too challenging.

With the damper in place, a large shift of the resonance frequency was observed when compared to the measurement without damper $(269 \mathrm{~Hz})$, as the OOP and IP linear resonance were at $435.9 \mathrm{~Hz}$ and $393 \mathrm{~Hz}$ respectively. The frequency response of the out of phase mode (OOP) in Fig.7a shows a significant amplitude reduction from the nearly linear case $\left(C F / F_{\text {exc }}=10000\right)$ to the higher excitation forces. This can be explained by the strong contribution of the local microslip on the energy dissipation mechanism as, even at low excitation levels, localised slip areas are present at the contact interface. A slight frequency shift can also be observed (less than 1\%) from the linear to the more damped measurements, but with the available excitation force $(17 N)$, no transition to an uncoupled motion was observable in the test range.

The IP mode (Fig. 7b), shows a slightly diminished amplitude, and in addition large jumps appear at low force ratios due to the strong nonlinear softening in the system. All the FRFs were measured with a down sweep, from high to low frequencies, as it allowed a better capture of the maximum response, following the upper branch of the 


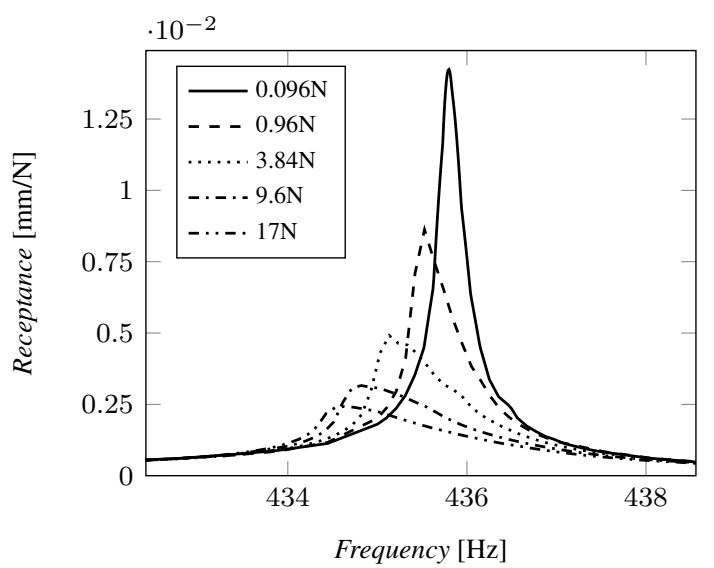

(a)

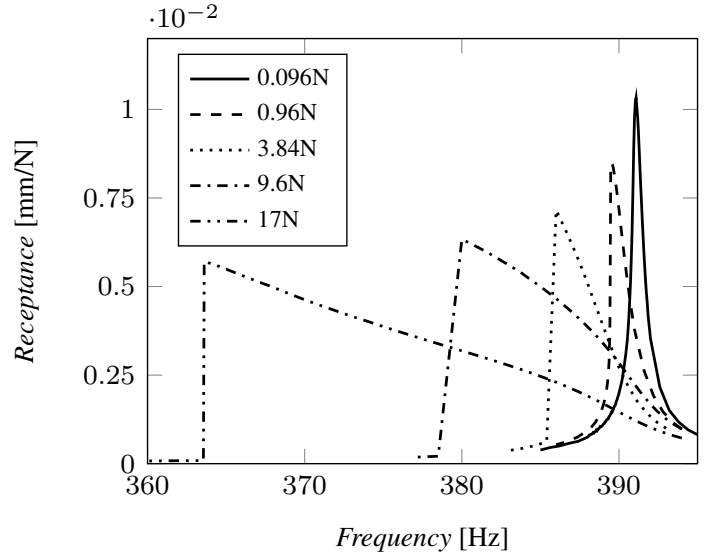

(b)

Figure 7: Experimental FRFs measured at a constant damper load (960 N) and increasing shaker excitation forces: a) first flexural OOP mode of the blades (down sweep: from high to low frequencies), b) first flexural IP mode of the blades (down sweep: from high to low frequencies).

overhanging FRF. The strong softening effect observed in the in phase FRFs (see Fig. 7b has a significant impact on the blade dynamics, as it causes large shifts of the resonance frequency compared to the linear case. A possible explanation for this much stronger nonlinear behaviour in the IP mode could be the tendency of the damper to roll [6, 32], which can reduce the damper-platform contact area at higher amplitudes, leading to a continuously changing and softer system.

To confirm the presence of a rolling motion of the damper, a local measurement of the damper kinematics was performed for the IP and OOP modes. Two LDV single-point lasers were directed by two mirrors to the bottom corners of the damper as shown in Fig. 8a similarly to the set up shown in [32]. It can be seen that for the IP mode, the bottom corners of the damper are moving out of phase, causing a rolling motion, whereas the OOP mode shows a pure translation of the damper (Fig $8 \mathrm{~b}$ ). This confirms the strong difference in damper motion between the IP and OOP mode, and will present a challenging test case for the explicit damper analysis.

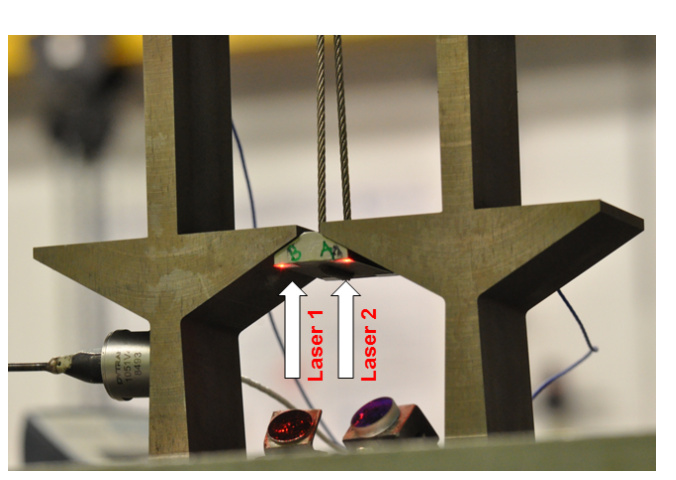

(a)

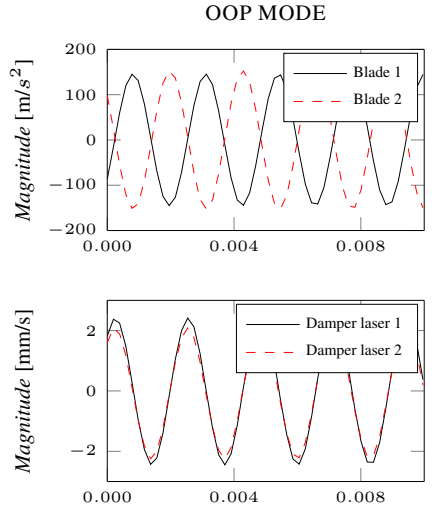

time $[\mathrm{s}]$
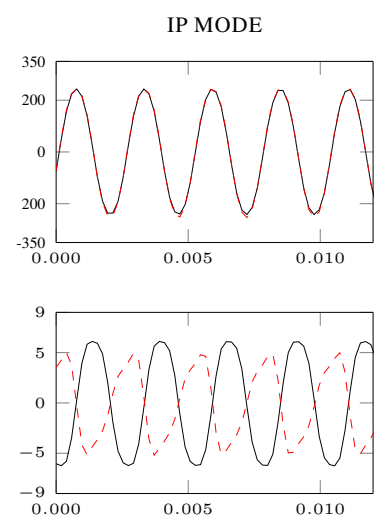

time $[\mathrm{s}]$

(b)

Figure 8: Measurement of the damper kinematics: a) laser measurement positions, b) damper motion (bottom plots) for the IP and OOP blades mode (top plots). 


\section{Simulations: influence of model parameters}

Before the optimum explicit model set up could be determined and compared to the experimental findings, a numerical investigation was performed to understand the influence of the input parameters on the dynamic response. In particular, as these input parameters used in the contact model are related to physical aspects of the real contact, gaining an understanding of their role could give insights on the physical mechanisms involved in the system dynamics. The influence of a non uniform contact pressure distribution is shown in section 5.1, and of the contact stiffness in section 5.2. Finally, the static term in the nonlinear force expansion, and its importance for damper modelling is discussed in section 5.3

\subsection{Contact pressure distribution}

In the UPD rig, the centrifugal force (CF) experienced by the damper in the engine is simulated by a pulley system, which loads the damper against the blade platforms. This might lead to an uneven pressure distribution at the interface, which is often neglected during the modelling phase and replaced by a constant value [20]. In this case, the initial pressure $\sigma_{0}$ on each side of the damper can be evaluated, taking friction in the contact into account, as follows:

$$
\sigma_{0}=\frac{1}{2} \frac{C F}{A(\cos (\alpha)+\mu \sin (\alpha))}
$$

where $C F$ is the radial force, $\alpha$ is the damper angle and $A$ is the contact area on each side. Particular care needs to be taken when using Eq 5 as it is only valid if the tangential forces equal the Coulomb friction forces, which was true for the specific damper geometry tested. However this assumption of a constant pressure represents a strong simplification, and different damper and platform shapes will lead to different pressure distributions at the contact interface. A pressure gradient with the highest level in the central area was observed experimentally in the UPD rig by inserting pressure films at the damper-blade interface as shown in Fig $9 \mathrm{a}$

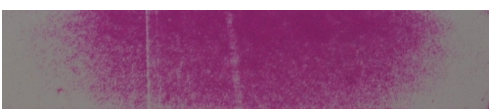

(a)

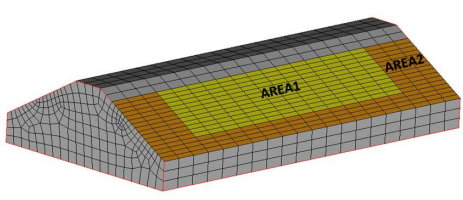

(b)

Figure 9: a) Pressure film print measured at the damper contact interface, b) FE model of the damper contact areas used for the pressure investigation.

To investigate how an uneven pressure distribution affects the dynamics of the system, a simplified model was built, where each contact area was split into two parts of uniform initial pressure as shown in Fig 9b The central area, referred to as "area 1" in Fig 9b, carries a higher load compared to "area 2", but the total load transmitted is constant and equal to $\sigma_{0} A$. Different distribution ratios of the load between these two areas were generated $(70 \%-30 \%, 80 \%$ $20 \%, 90 \%-10 \%, 100 \%-0 \%$ ) and the FRFs at various excitation levels under a constant pulling load (960 N) were computed. The 1F OOP mode was selected for the parameter study, as it allows faster calculations when compared to the IP mode, due to a better convergence of the results. The results at two different excitation levels were computed, $3.84 \mathrm{~N}$ (250 force ratio) in Fig 10a and $17 \mathrm{~N}$ (56 force ratio) in Fig $10 \mathrm{~b}$ The lower value was chosen because a significant nonlinear behaviour was starting to be observed experimentally at this level (see Fig[7a), whereas $17 \mathrm{~N}$ represented the maximum excitation achievable by the shaker.

Fig 10a shows that a more "uneven" distribution leads to higher energy dissipation for the 1F mode at $3.84 \mathrm{~N}$ excitation level. A delta in amplitude of $65 \%$ can be observed compared to the case when the load is uniformly 


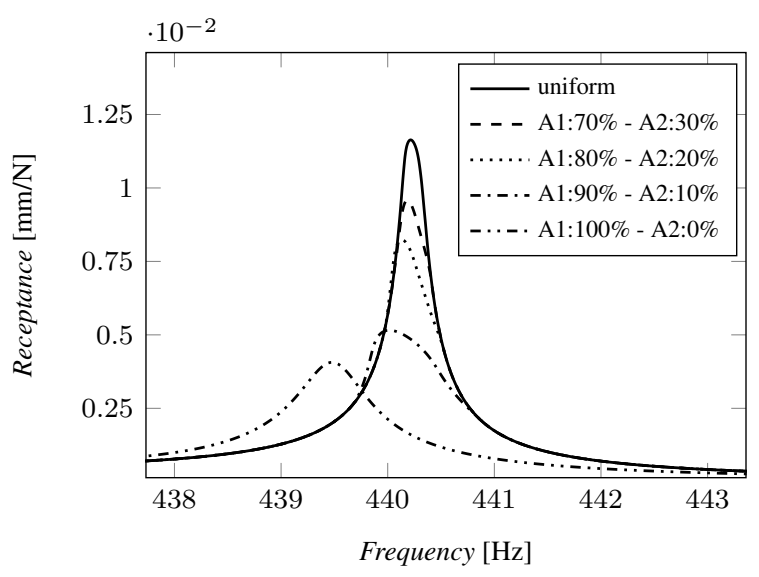

(a)

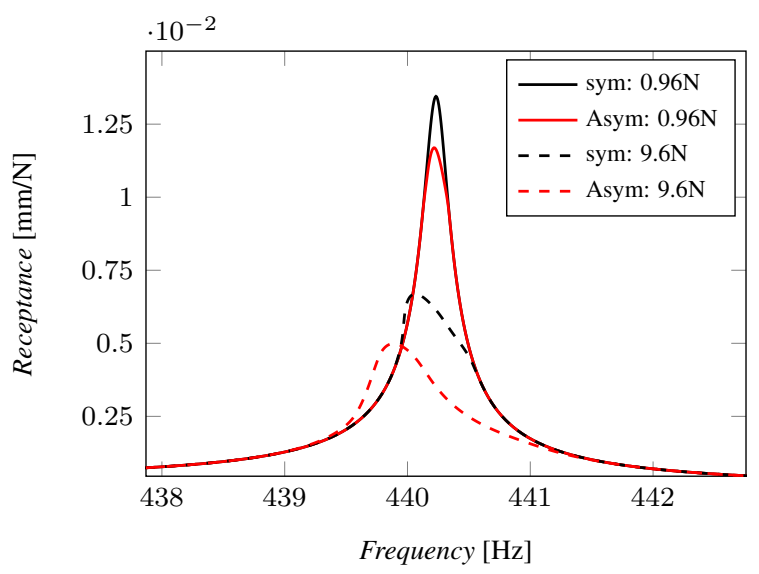

(c)

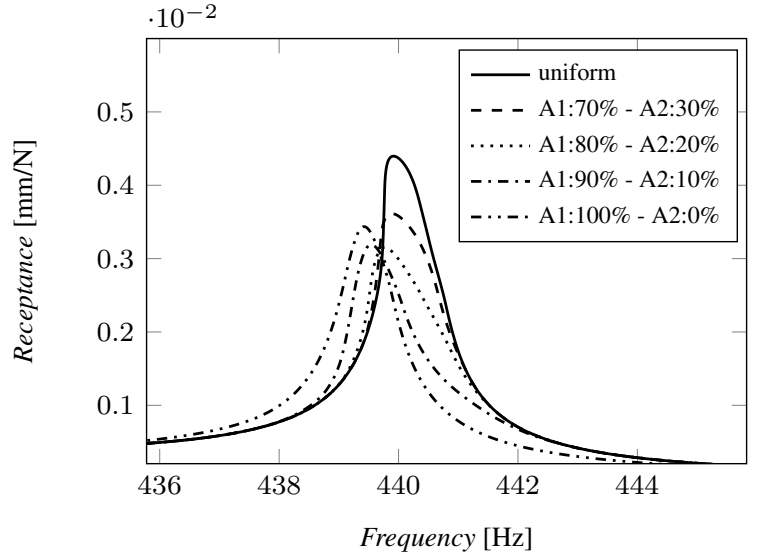

(b)

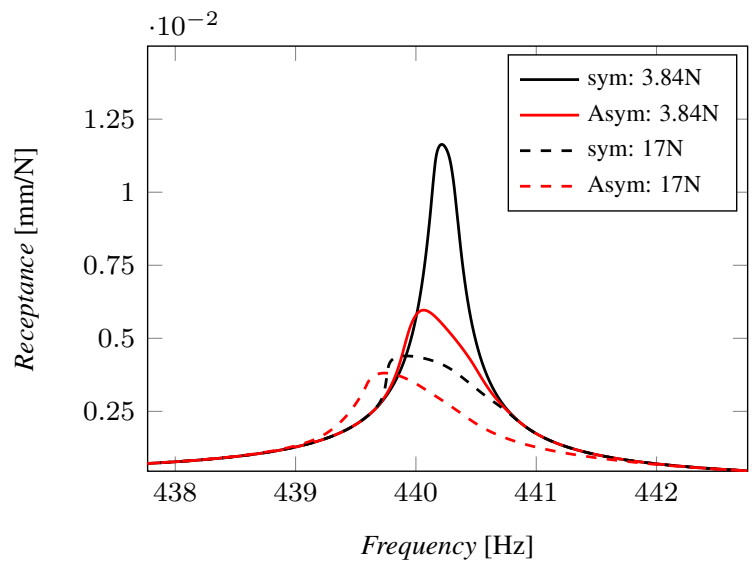

(d)

Figure 10: Simulated FRFs using different pressure distribution between the two areas of the damper interface. a) Effect of various distributions at $3.84 \mathrm{~N}$ excitation force, b) effect of various distributions at $17 \mathrm{~N}$ excitation force, $\mathrm{c}, \mathrm{d}$ ) simulated FRFs with uniform and asymmetric pressure distributions and various excitation forces.

distributed among area 1 and 2. At higher excitation levels ( Fig $10 \mathrm{~b}$ ), the sensitivity of the change in amplitude is less dependent on the distribution ratio, which seems to indicate that when the contact interface is closer to a macroslip regime (high amplitudes), its dynamic response is less sensitive to variation in the distribution. This can be explained as the nonlinear behaviour of the contact elements that carry a lower initial pre-load is activated at lower excitations compared to the highly loaded ones. If the excitation is then increased significantly, all the contact elements are triggered into a similar slip regime uniformly, regardless of their initial static condition. Therefore a highly non-uniform pressure distribution, commonly observed in mechanical joints, leads to a smoother transition from linear dynamics to nonlinear dynamics governed by a macroslip beahviour when the harmonic excitation is increased. In addition, a minimum peak amplitude appears in Fig $10 \mathrm{~b}$ for the A1:80\%-A2:20\% distribution, showing an optimal distribution for that particular excitation level. A further decrease of the load in the A2 area leads to less damping, which indicates that some energy dissipation is lost when A2 becomes unloaded at high excitation level. A slight change in resonance frequency can be observed as well with different pressure distributions, but it is rather insignificant compared to the strong frequency shift caused by the presence of the damper. A relatively common loading of UPDs in an engine is an asymmetric load, where one side will be under much more loading than the other side, potentially leading to one stuck and one sliding contact surface. The damper will be loaded and unloaded at least once during each flight cycle due to the centrifugal force, and it may end up in a slightly different position, leading 
to such asymmetric loading. To explore the effect of an asymmetric settling of the damper or a geometric difference between the two sides, a simulation was run using a uniform distribution on one side and an uneven one on the other side (A1:90\%-A2:10\%). The results reported in Fig.10c and Fig 10d highlight the impact of these asymmetric sides on the blade dynamics, particularly evident at lower excitation levels. The observed sensitivity of the nonlinear dynamic response, implies that a good description of the pressure distribution at the interface is important when accurate predictions are required.

\subsection{Contact stiffness}

In the contact model used for the analysis (Fig $6 \mathrm{~b}$ ), the tangential and normal stiffnesses describe the elastic properties of the contact interface. The tangential stiffness was measured experimentally as described in [42], leading to an average value per unit area of about $60000\left[\mathrm{~N} / \mathrm{mm}^{3}\right]$. The corresponding normal stiffness was chosen to be the same, as in a rough dry contact the same order of magnitude is expected [43], and no measured values were available. It should be noticed that this assumption constitutes a simplification since the real ratio between the two stiffnesses depends on the geometry of the contact patch [44], but previous experience [45] has shown that it does not affect significantly the results. In addition, as each contact element at the friction interface is associated with a patch of different geometry (which comes from the meshing process of the interface), this would lead to difficulties, as it would require defining a different contact ratio for each of them. Both tangential and normal stiffnesses depend on the proximity of the asperities at the interface, and their values increase with the load ([44]), but the current nonlinear friction model (see section 3.2) does not allow such a behaviour, so that they were assumed to be constant. In addition, during the measurements, some scatter was observed for the tangential stiffness values and an average value was selected. It is therefore interesting to understand the effect of different values of the contact stiffness on the nonlinear dynamics of the blade, and for this reason, a numerical investigation was carried out using a large range of stiffnesses from $5 k\left[\mathrm{~N} / \mathrm{mm}^{3}\right]$ to $10000 k\left[\mathrm{~N} / \mathrm{mm}^{3}\right]$. The main effect of the variation of these values is a slight shift (around 1\%) in the resonance frequency, which starts to be more evident at the lowest stiffness values (see Fig 11a and Fig $11 \mathrm{~b}$. An increase in the amplitude of the nonlinear response can also be observed for lower stiffness values, which can be attributed to the extended linear range of some contact elements, which do not reach slip state. These findings indicate that the sensitivity of the model to a change in contact stiffness is relatively low with respect to the frequency, but a possible inaccuracy in the nonlinear amplitude estimation can arise at lower excitation levels.

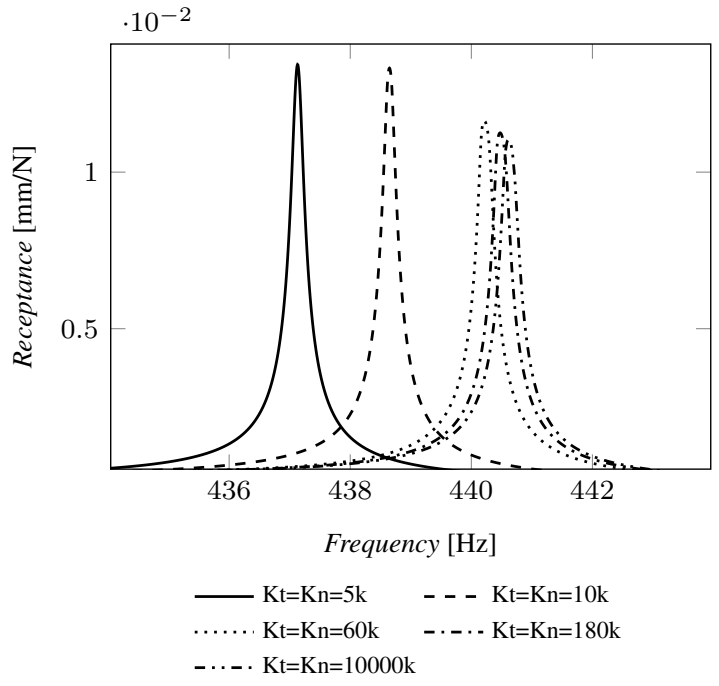

(a)

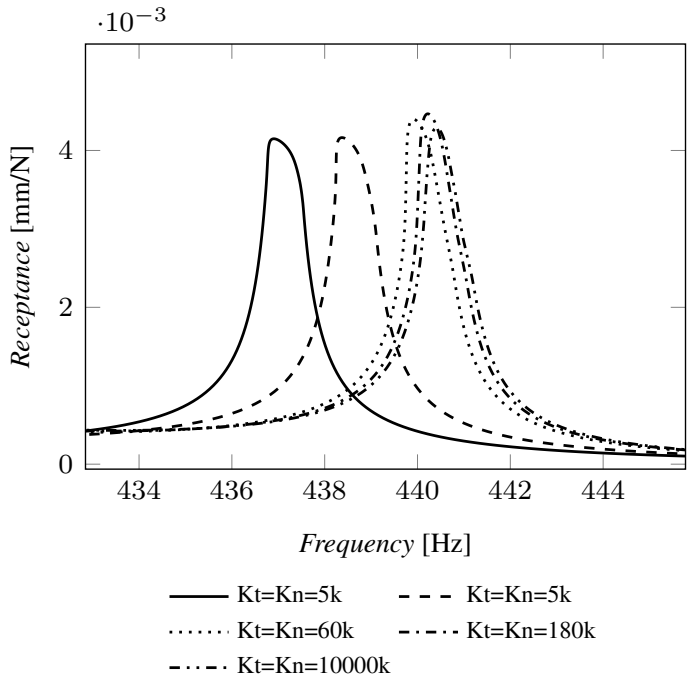

(b)

Figure 11: Simulated FRFs using various normal and tangential contact stiffness for the contact model. a) Effect of various contact stiffness at 3.84 $\mathrm{N}$ excitation force, $\mathrm{b}$ ) effect of various contact stiffness at $17 \mathrm{~N}$ excitation force. 


\subsection{Dynamic loading and harmonic "zero"}

For this study, a decoupled approach, which separates the static and dynamic problems, was chosen for a better convergence of the numerical solution and to allow different levels of detail for the static calculation. This means that the static load at the damper interface caused by the centrifugal force are calculated independently from the dynamic analysis. This static load can be either assumed to be uniform, can be calculated with an FE contact analysis or via interpolation of experimental measurements (as shown in section 6). The decision to keep the static problem decoupled guarantees a higher flexibility during the study, as different ways to evaluate the static contact problem (pressure films, FE contact analysis, semi-analytical methods) can be used, keeping the dynamic model always the same. For each contact element, an initial pre-load N0 (static load) is used to calculate the tangential and friction forces. The input force used for the nonlinear dynamic analysis is single harmonic to reproduce the shaker excitation, while the response is approximated with a multi-harmonic expansion.

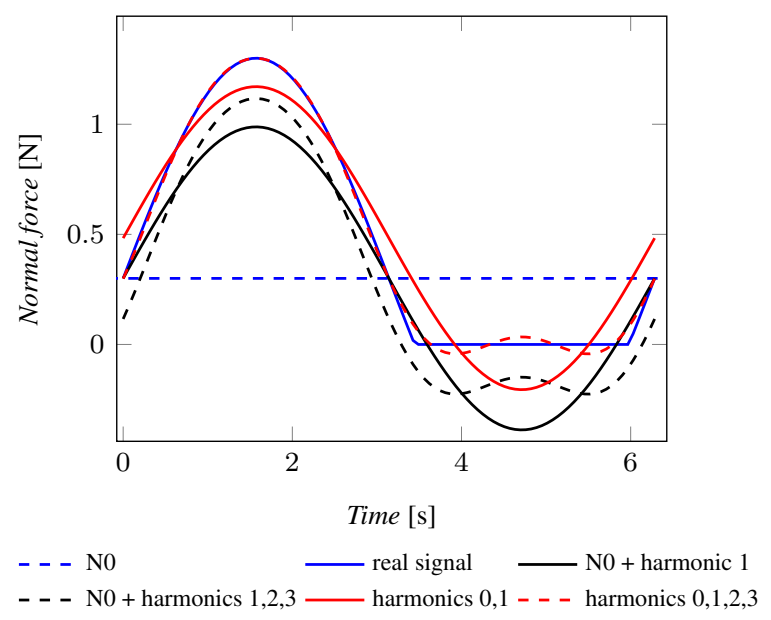

(a)

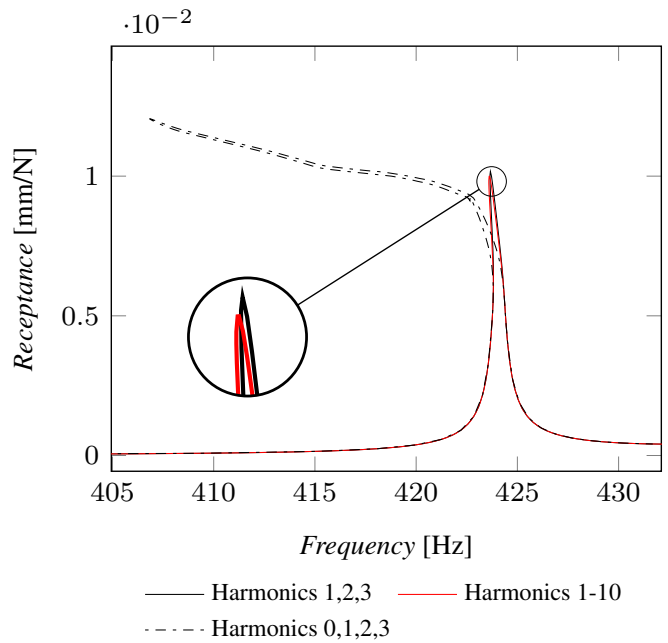

(b)

Figure 12: Investigation on the effect of different harmonics used for the multi-harmonic expansion: a) different harmonics used to approximate a simulated normal force signal with a separation event, b) simulated FRFs with different number of harmonics.

Due to the damper kinematics, significant variation of the normal load occurs during a vibration cycle. To visualise this problem, a simplified example is shown in Fig.12a, where the blue signal represents a "real" normal load oscillation with separation during the cycle. If only the first three harmonics are included in the approximation and the static term N0 from the decoupled static analysis is then added, the simulated signal is not able to replicate the original one. A good approximation of this force signal can be obtain only when the harmonic "zero" term (constant term) is included in the expansion. For the UPD test rig, this harmonic "zero", which appears only at higher vibration amplitudes, allows a change of the mean vibrating position of the blades and the damper during a response calculation. In particular, when approaching the resonance frequency the damper tends to gradually move higher, and the blades tend to open leading to a new mean position, as shown in Fig 13

The consequence of this change on the blade dynamics is fundamental, as can be seen in Fig $12 \mathrm{~b}$ where the system response is simulated including harmonics 1 to 3,1 to 10 and 0 to 3 . The simulation with 3 and 10 harmonics almost overlap, indicating that the higher harmonics do not contribute to capture the response any better, whereas the curve obtained with the zero harmonic term differs significantly and shows a marked softening behaviour. Here the 1st flexural IP mode is reported, as it is more affected by the normal load variation at the contact interface. In summary, when modelling under platform dampers, the harmonic "zero" term plays a key role to determine the dynamic response accurately, as the system response shows a great sensitivity to a change of the mean vibrating position. It should be noted at this point, that this effect is probably particularly strong for the investigated configuration, since in a real engine configuration the blades may not be able to separate so strongly, since the adjacent dampers will restrict the opening of the blades. 


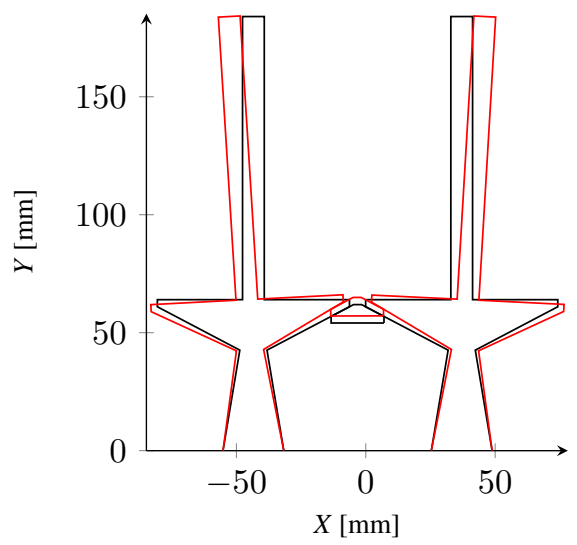

Figure 13: Change of the mean equilibrium position when approaching the IP resonance during a simulated response. The scale of the plots has been magnified (400x) to allow a better visualisation.

\section{Simulations: updated model}

Based on the findings of the previous section, a final model which includes more realistic model assumptions for the UPD rig was developed, and the response for different excitation levels was computed for the 1F IP and OOP modes to allow a comparison against the measured data.

\subsection{Experimental and numerical comparison}

Given the importance of the correct pressure at the interface, as demonstrated in section 5.1, a static FE analysis was initially carried out to compute the static contact conditions. The resultant pressure distribution from the FE analysis showed a strong stress concentration in the areas around the bottom edge of the damper, which did not correspond to the pressure films prints (see Fig $14 \mathrm{~b}$ ) and can be explained by the difference in the shape of the damper.

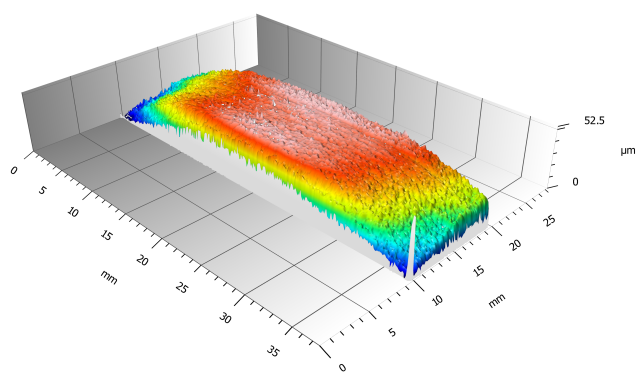

(a)
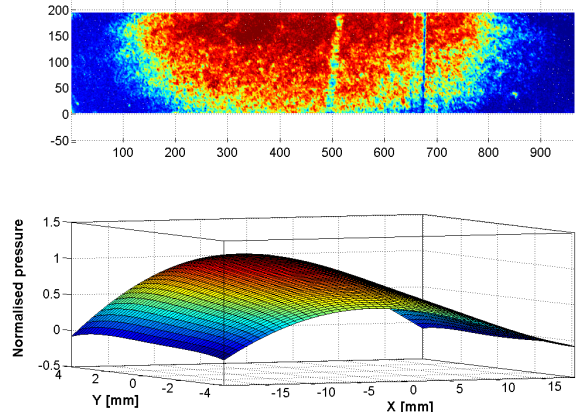

(b)

Figure 14: a) Experimental damper 3D surface profile obtained with a scanning interferometer, b) pressure film prints at the damper interface acquired and fitted with a polynomial surface in MATLAB.

A 3D surface profile of the real damper was performed with a Polytec TMS100 interferometer, and revealed that both sides were slightly curved as a consequence of the manufacturing process (see Fig 14a). To understand the impact of these profiles on the pressure distribution, a Fuji Prescale pressure film was used at the damper-platform contact, showing a strong increase in pressure around the apex of the curvature on each side. Since it proved to be 
challenging to reproduce the correct profile geometry in the FE model, the imprints of the pressure films were scanned and digitised in MATLAB, and their values used as an initial condition for the nonlinear dynamic analysis. The print scan of one side converted to an intensity image is shown on the top of Fig $14 \mathrm{~b}$. An area of progressive higher pressure is visible near the top center of the damper, corresponding to the profile of the damper. A $4^{\text {th }}$ order polynomial was then fitted to the intensity image to have an approximated pressure distribution, which was then interpolated onto the contact nodes to obtain the initial pre-load (Fig 14b for the nonlinear dynamic analysis. It should be noticed that the pressure film itself represents an additional elastic layer leading to potential uncertainties in the pressure readings, and for this reason, a re-scaling process was performed on the pressure distribution to match the total transmitted load (known from Eq 5].

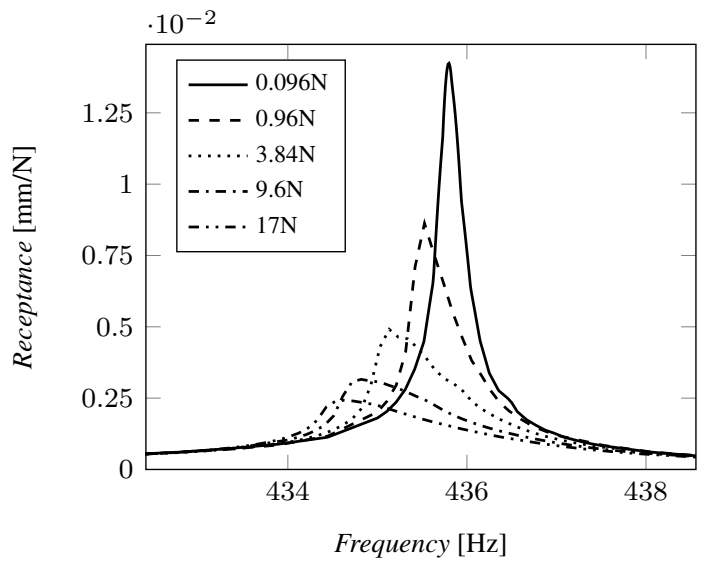

(a)

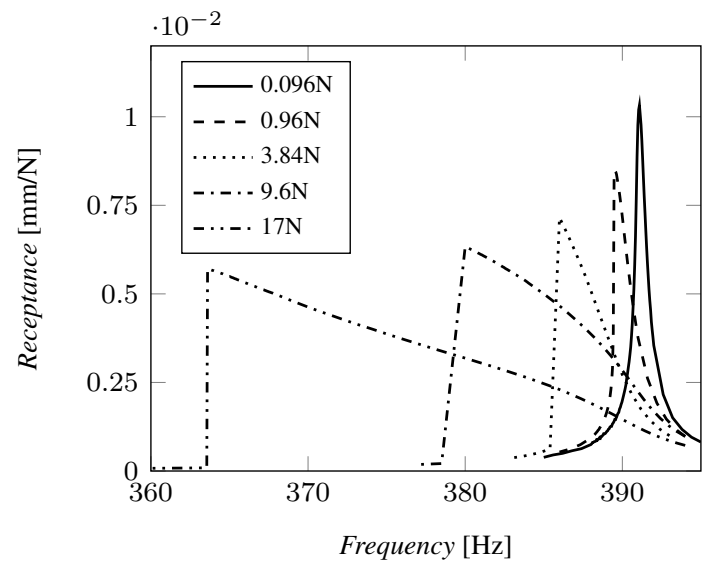

(c)

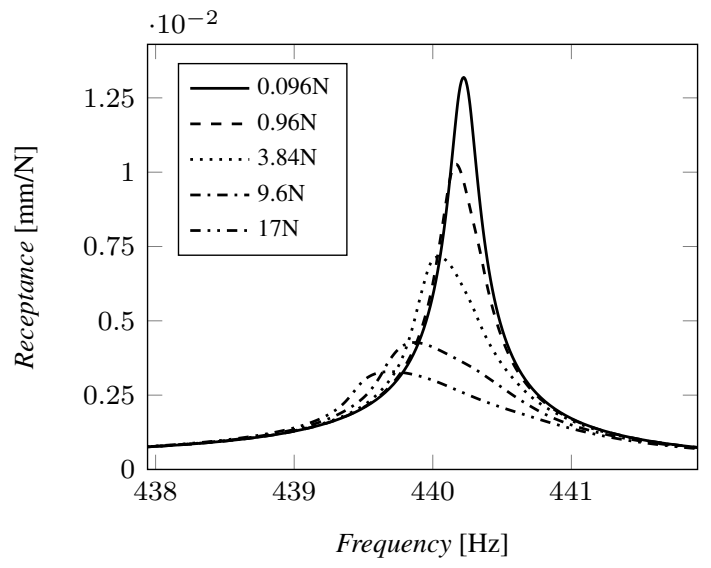

(b)

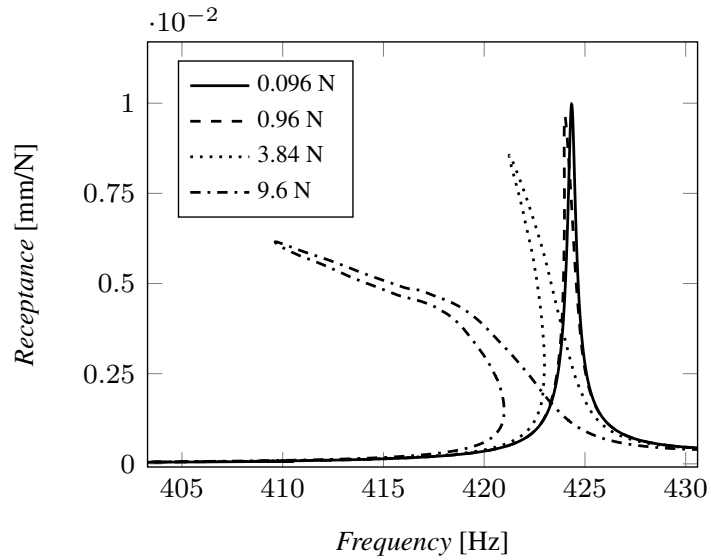

(d)

Figure 15: Comparison of the measured and simulated FRFs for both modes at different force levels: a) experimental FRFs OOP mode, b) simulated FRFs OOP mode, c) experimental FRFs IP mode, d) simulated FRFs IP mode.

The friction coefficient $\mu=0.6$ and a tangential, $K_{t}$ and normal $K_{n}$ contact stiffness of $60000 \mathrm{~N} / \mathrm{mm}^{3}$, were based on previously obtained experimental data [42]. The harmonic "zero" was included in the nonlinear force expansion due to its importance for the IP modes, and the first three non zero harmonics were used, as they were shown to be adequate to capture the effect of local friction and separation on the blade dynamics correctly (see section 5.3). The resulting experimental and numerical FRFs of the OOP and IP 1st flexural mode are shown in Fig. 15 for different force ratios ranging from the linear case (excitation $0.096 \mathrm{~N}$ ) to $17 \mathrm{~N}$, which is the maximum excitation force tolerated by the shaker. The predicted resonance frequency with the damper in place are very accurate for the OOP mode (about 
$1 \%$ difference), but they are about $8 \%$ higher for the IP mode. A possible explanation could be the assumption of a constant $K_{t}$ and $K_{n}$ with regards to pressure, as it could be more limiting for the IP mode, which involves rotation of the damper and consequently significant normal load variation. The slight frequency shift of the OOP mode at higher excitation levels (Fig 15a) seems to be reproduced correctly, and so is the strong nonlinear softening effect observed experimentally in the IP mode (see Fig $15 \mathrm{c}$ and Fig $15 \mathrm{~d}$ ). In a real engine, a good approximation of this softening effect is important as it could lead to higher amplitudes (and stresses) for a larger operating range, as well as a change in the apparent resonance frequency measured depending on the sweep direction.

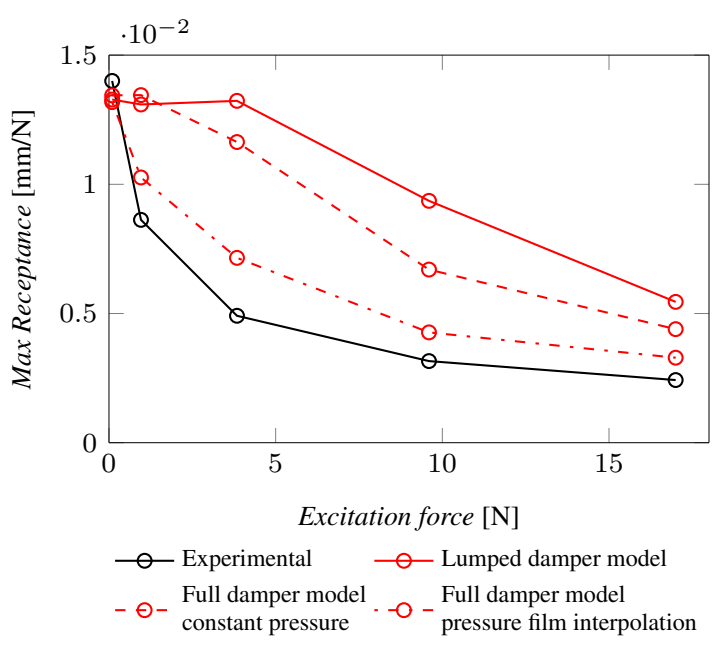

(a)

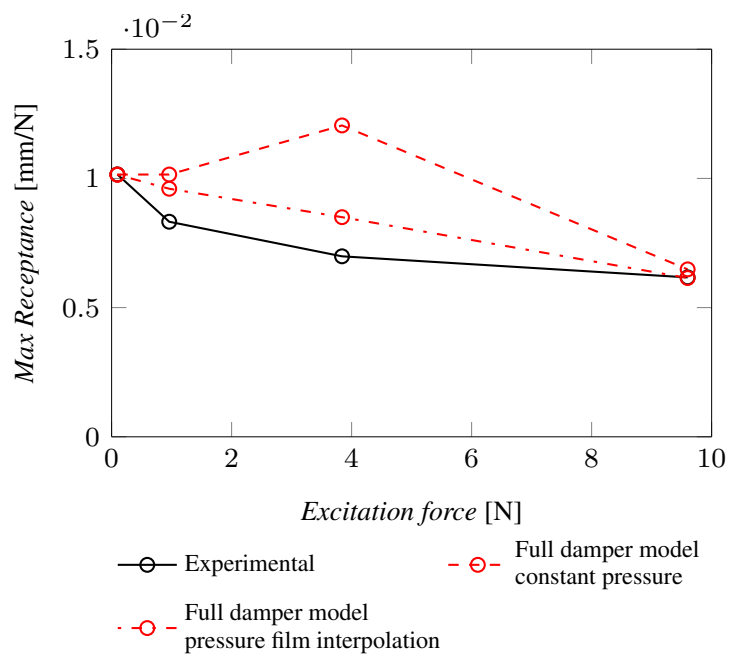

(b)

Figure 16: Peak amplitude comparison between the measured and simulated FRFs at increasing force levels: a) amplitude reduction OOP mode, b) amplitude reduction IP mode.

The experimental damper is very effective in reducing the vibration amplitude for the OOP mode (see Fig.16a), leading to an initial strong reduction in amplitude at low forcing levels, and a nearly constant low amplitude at maximum forcing levels. It is interesting to observe that the test rig with the available shaker excitation does not reach the state of uncoupling of the two blades that has been reported in other parts of the literature [6, 11] probably due to the limited force available. For the IP mode, the loss of contact during the rotation of the damper limits the energy dissipation capability, and leads to a more or less constant peak amplitude at large excitation levels (Fig 16b).

To evaluate the capability of the different damper models (explicit damper with constant pressure, explicit with pressure film prints and lumped mass model) to capture the experimental behaviour, the maximum amplitudes at different excitation levels are compared to the measured ones. The lumped mass damper with two contact elements, tends to overestimate the response in the OOP mode, especially at lower excitation levels. In fact, a single contact element on each platform, can not capture localised dissipation at the interface. For the IP mode, the lumped model, which can not capture the damper rotation, is linear in the force range explored, and for this reason is not reported in Fig $16 \mathrm{~b}$ The explicit damper model with a uniform pressure distribution leads to an under prediction of the damping in the IP and OOP case, where especially the initial sharp drop in amplitude of the OOP mode can not be captured with the model due to a delayed start of microslip at the contact edges. An additional interesting feature in the "constant pressure" predicted response of the IP mode is a rise in amplitude at medium excitation levels, which is due to the strong softening and consequently higher response caused by the damper rotation, and a failure of the model to predict the dissipated energy correctly. When the pressure film interpolation is used as an input, a better agreement can be achieved over the full range. The analysis now captures both the absolute amplitude and force dependence with good accuracy, showing that only an explicit model that is very close to reality can predict the complex nonlinear mechanisms at the interface correctly. 


\subsection{Damper kinematics and contact plots}

The strong influence of the zero harmonic and the static damper load indicate that a relatively complex motion is occuring at the contact interface. The damper is a floating body, and its spatial motion is the result of the interaction of the nonlinear forces generated at the contact interface and its own inertia properties. Given the similar global dynamic response of the simulations and the experiments (see last section), it was interesting to see whether the predicted damper motion would also match the observed one from $8 \mathrm{~b}$ in section 4 For this purpose, the harmonic coefficients of the displacements were extracted for several points lying on the front section of the blades. The 2D motion is visualised by expanding the multi-harmonic coefficients in the time domain for all the selected points, and by plotting the peak values for the OOP (Fig $17 \mathrm{a}$ ) and IP (Fig $17 \mathrm{~b}$ ) mode. The plots match the experimental findings, where a pure translation was observed for the OOP mode and a rotation occured in the IP mode. For the latter mode, the contact area on the platform that is lifting tends to shift down close to the lower edge and the contact on the platform which is lowering tends to move up close to the upper edge.

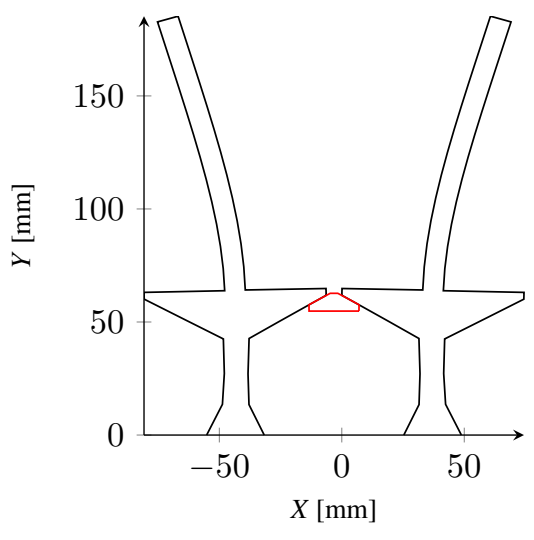

(a)

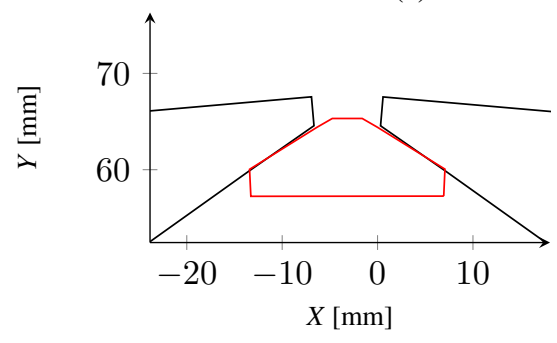

(c)

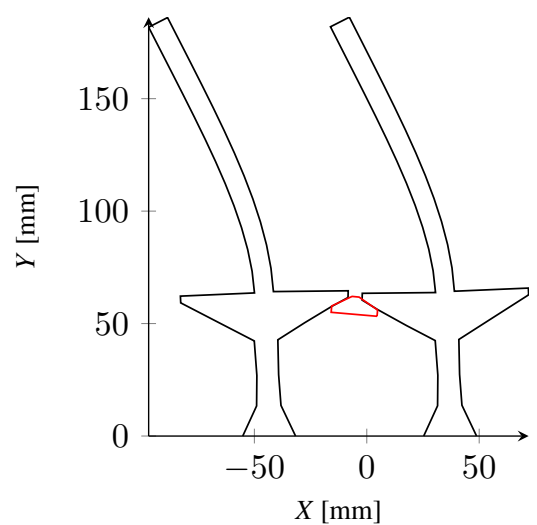

(b)

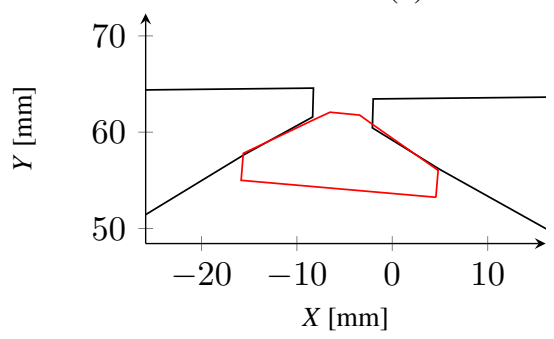

(d)

Figure 17: Simulated deflection shape of the blades and damper for both modes: a) simulated OOP mode, b) simulated IP mode, c) damper kinematics zoom OOP mode, d) damper kinematics zoom IP mode. The scale of the plots has been magnified to allow a better visualisation.

Moving one level closer to the interface, the large number of contact elements between the damper and the platform allows to obtain detailed information of the local contact dynamics that drives the observed behaviour. Each contact node (see Fig 18) can be classified into one of four different categories, depending on the conditions experienced during a vibration cycle: linear nodes that do not dissipate energy (blue dots in the plots), nodes undergoing stick-slip transition (green dots), nodes experiencing a separation event during the vibration cycle (red dots) and nodes that never get in contact (light blue dots). Fig 18 shows the contact conditions for the IP and OOP modes at low and large excitation forces. At low excitation forces a large number of stuck (linear) elements is present at the contact interface, but with increasing forcing, the number of elements that start to slip or separate increases. The elements far from the center start to slip earlier due to the measured lower initial static pressure at the edges. For the OOP mode, even at $17 \mathrm{~N}$ excitation force, a large number of elements is still stuck in the centre of the damper, confirming the experimental observation that the damper operates closer to the fully stuck than free condition. The detailed contact plots also help to explain the strong FRFs softening observed in the IP mode. The large area of stuck elements at 


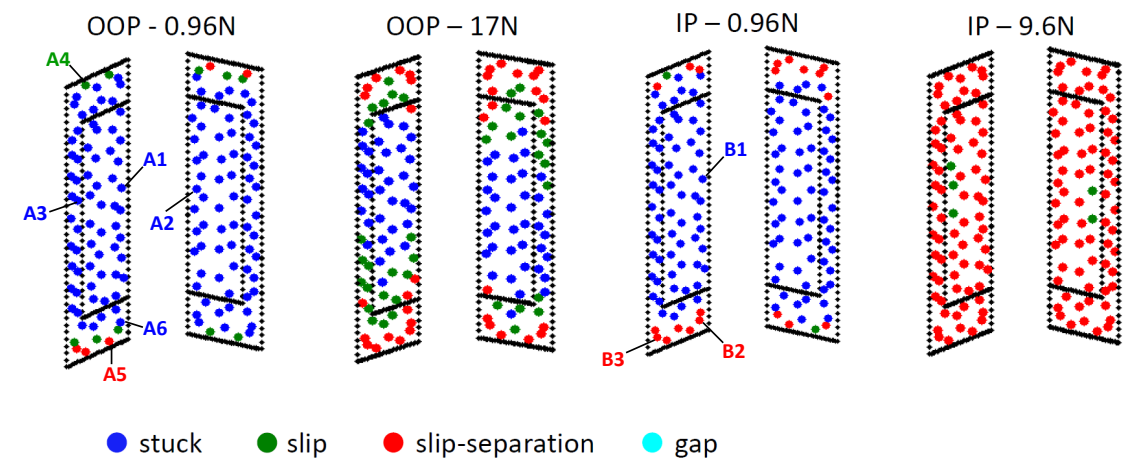

Figure 18: Contact conditions for each contact node at the damper interface. Different levels of excitation are shown for the OOP and IP mode.

$0.96 \mathrm{~N}$ excitation entirely disappears at the higher levels where almost all the elements experience separation at one point during the vibration cycle. When this occurs, the coupling between the two blades tends to become weaker and the resonance frequency moves towards the frequency of the free blades. Fig 19 shows the evolution of the local tangential and normal forces for a subset of nodes, at the contact interface allowing a more detailed understanding of the underlying nonlinear mechanism that drives the behaviour of the UPD. It can be observed that nodes A1, A2, A3, A6 and B1 are stuck at all times as their tangential forces are below the friction limits (red dotted lines) and the normal forces are positive. A1 and A2 have the same phase, confirming the symmetry of the interface motion for the OOP mode as observed in Fig 17a Node A1 and A3 show a similar phase for the tangential force, but the normal forces are out of phase, indicating a rotation around an axis located near the centre of the damper and along its main dimension. Moving closer to the edge, the contact pressure becomes lower (see node A6) and the tangential force almost reaches the friction limit during a vibration cycle. Node A4 undergoes a stick-slip transition with the tangential forces following the friction limit for large part of the vibration cycle, leading to significant energy dissipation. In node A5, an initial separation occurs at the beginning of the cycle, followed by a phase of contact, during which slip is observed. For the IP mode, nodes B2 and B3 close to the edges show an out of phase separation event confirming the measured rotation of the damper. Having access to detailed information about the state of the contact elements and their evolution over time helps to understand the dissipation mechanism and may lead to a more locally optimised damper geometry in the future.

\section{Conclusions}

A new underplatform damper test rig was designed and commissioned to validate the prediction capabilities of highly detailed explicit damper models. A simplified static set-up with two pseudo blades was chosen, and a set of available and newly-defined nondimensional parameters were used to ensure a dynamic behaviour similar to a real HPT blade. The investigation focused on the 1 st flexural in phase (IP) and out of phase (OOP) modes, since they show large amplitudes which can cause high alternating root stresses.

Different forcing levels highlighted a strong energy dissipation for the experimental OOP mode, which could be attributed to microslip friction dissipation, whereas the IP mode showed a softening effect on the FRFs caused by the rotation of the damper. The latter was confirmed by local measurements of the damper kinematics.

A nonlinear model of the test rig was generated, based on a three dimensional FE model of both blades and damper, which were coupled through a dense grid of 3D friction contact elements. An initial parameter study identified the static contact pressure distribution as the most influential parameter on the dynamic response, making its accurate 

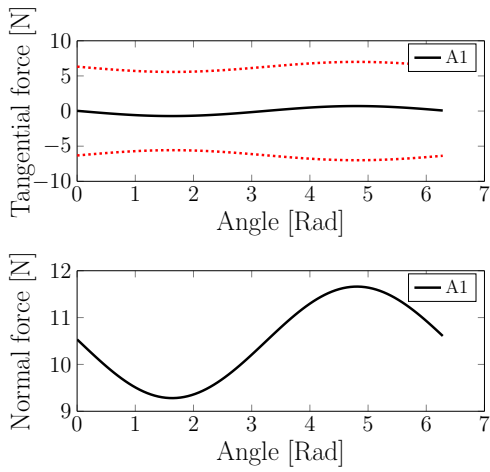

(a)
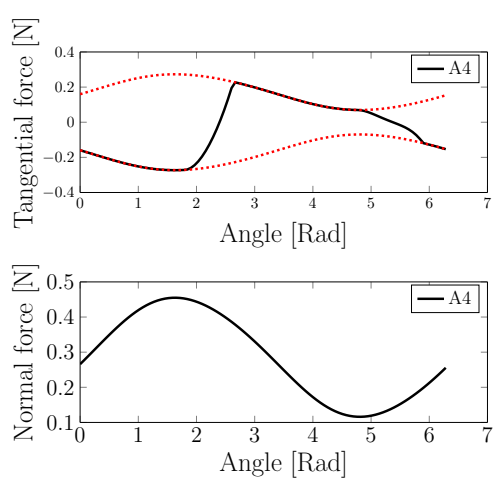

(d)
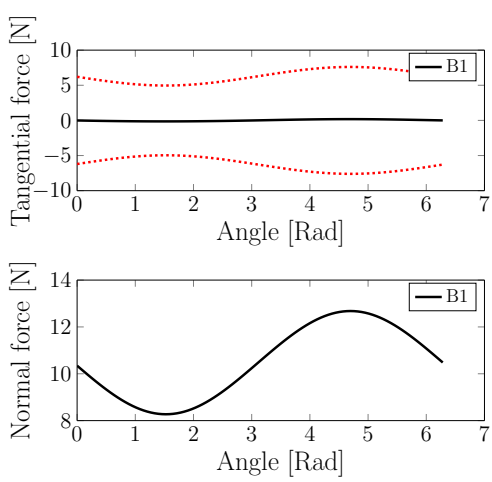

(g)
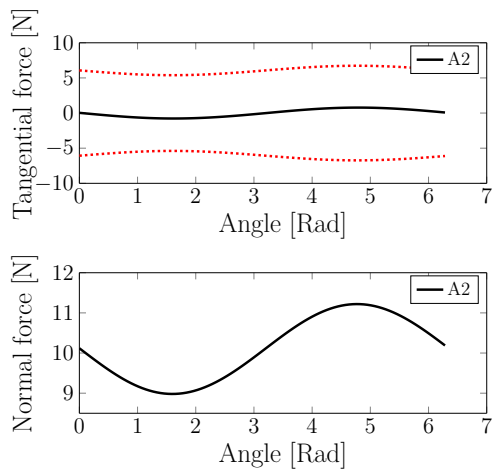

(b)
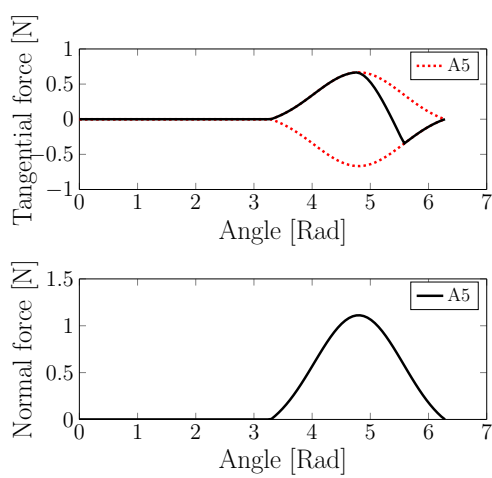

(e)
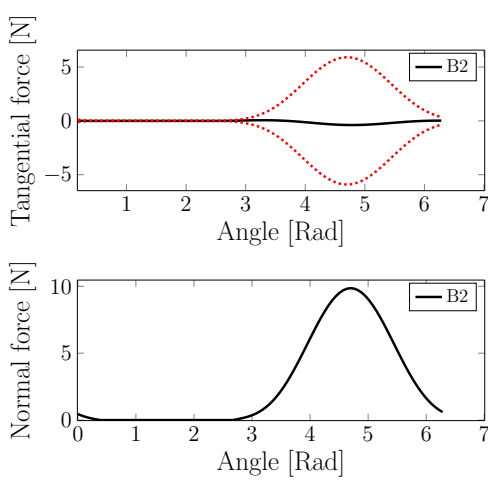

(h)
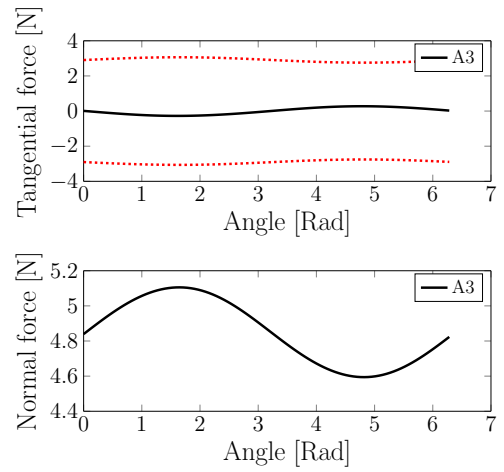

(c)
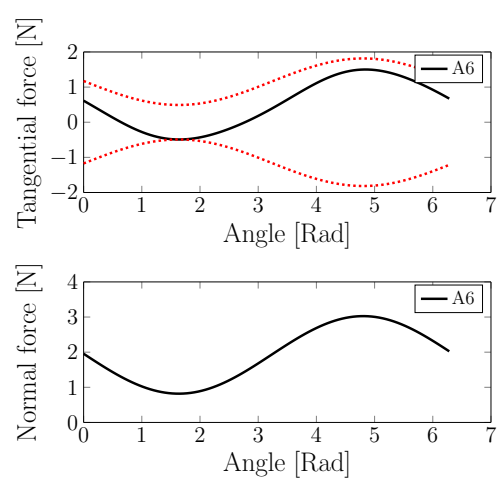

(f)

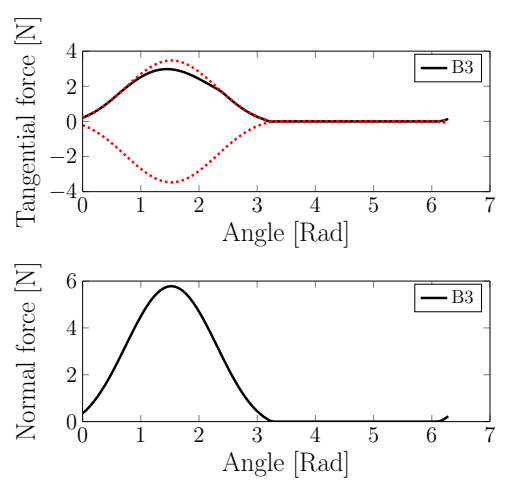

(i)

Figure 19: Contact force evolution for different location points: a) node A1, b) node A2, c) node A3, d) node A4, e) node A5, f) node A6, g) node $\mathrm{B} 1, \mathrm{~h})$ node $\mathrm{B} 2, \mathrm{i})$ node $\mathrm{B} 3$.

capture in the model a high priority. An additional sensitivity was observed with regards to the element contact stiffness, impacting both the resonance frequencies and the amplitude response. When a large normal load variation occurred at the interface during a vibration cycle, as in the 1F IP mode, the "zero" harmonic term (constant term) was found to be essential for capturing the correct blade dynamics. In fact the increase in value of this static term near the blade resonance caused a change of the mean position of the blades, which, together with the damper rolling, led to a strong softening of the system.

The final model of the test rig, based on a measured pressure distribution from the real damper interface, measured 
friction coefficient and contact stiffness values, and the 0-3rd harmonic, showed a good agreement with the experimental data at different excitation levels. The model was able to capture localised dissipation caused by microslip effects, as well as the IP mode FRF softening, which highlights its ability to capture strong nonlinear phenomena such as separation over a large area of the contact interface.

The damper kinematics were reconstructed from the simulations, showing a damper translation for the OOP mode and a damper rotation for the IP mode, confirming the experimental findings. A classification of the contact elements based on their contact condition during a vibration cycle was used to visualise the local joint dynamics, gathering information about possible wear or separation zone and their evolution with the excitation force.

Predicting correctly the nonlinear behaviour of bladed disks constrained by underplatform dampers is a challenging task for turbine design. The detailed explicit damper model, with an improved description of the contact interface presented in this paper, proves to be effective in predicting the experimentally-observed nonlinear phenomena caused by dampers. In addition to this, the explicit damper model allows the damper kinematics and the local contact condition to be reconstructed, providing additional information which can be used during damper design to optimise the geometry and predict wear areas.

\section{Acknowledgments}

The authors are grateful to Innovate UK and Rolls-Royce plc for providing the financial support for this work and for giving permission to publish it. This work is part of a collaborative R\&T project SILOET II P19.6 which is co-funded by Innovate UK and Rolls-Royce plc and carried out by Rolls-Royce plc and the Vibration UTC at Imperial College London.

\section{References}

[1] E. P. Petrov, D. J. Ewins, State-of-the-art dynamic analysis for non-linear gas turbine structures, Proceedings of the Institution of Mechanical Engineers, Part G: Journal of Aerospace Engineering 218 (3) (2004) 199-211. doi:10.1243/0954410041872906

[2] B.A.Cowles, High cycle fatigue in aircraft gas turbine - an industry prospective, International Journal of Fracture 80 (1996) 147-163. doi : $10.1007 / \mathrm{BE} 00012667$

[3] L. Gaul, R. Nitsche, The Role of Friction in Mechanical Joints, Applied Mechanics Reviews 54 (2) (2001) 93-106. doi:doi:10.1115/ 1.3097294

[4] B. Feeny, N. Hinrichs, K. Popp, A historical review on dry friction and stick-slip phenomena, Applied Mechanics Reviews 51 (5) (1998) 321-341.

[5] J. H. Griffin, A review of friction damping of turbine blade vibration, International Journal of Turbo and Jet Engines 7 (1990) $297-307$.

[6] K. Y. Sanliturk, D. J. Ewins, A. B. Stanbridge, Underplatform Dampers for Turbine Blades: Theoretical Modeling, Analysis, and Comparison With Experimental Data, Journal of Engineering for Gas Turbines and Power 123 (4) (2001) 919. doi:10.1115/1.1385830

[7] K. Y. Sanliturk, D. J. Ewins, R. Elliott, J. S. Green, Friction Damper Optimization: Simulation of Rainbow Tests, Journal of Engineering for Gas Turbines and Power 123 (4) (2001) 930. doi:10.1115/1.1391278

[8] J. H. Griffin, Friction Damping of Resonant Stresses in Gas Turbine Engine Airfoils, Journal of Engineering for Power 102 (1980) $329-333$.

[9] G. Csaba, Forced Response Analysis in Time and Frequency Domains of a Tuned Bladed Disk With Friction Dampers, Journal of Sound and Vibration 214 (3) (1998) 395-412. doi:10.1006/jsvi.1997.1513

[10] B. D. Yang, C. H. Menq, Characterization of Contact Kinematics and Application to the Design of Wedge Dampers in Turbomachinery Blading : Part 1 Stick-Slip Contact Kinematics, Journal of Engineering for Gas Turbines and Power 120 (April 1998) (1998) $410-417$.

[11] L. Panning, W. Sextro, K. Popp, Optimization of interblade friction damper design, in: Proceedings of ASME TURBOEXPO, 2000 , pp. 1-8.

[12] J. Szwedowicz, C. Gibert, T. P. Sommer, R. Kellerer, Numerical and Experimental Damping Assessment of a Thin-Walled Friction Damper in the Rotating Set-Up With High Pressure Turbine Blades, Journal of Engineering for Gas Turbines and Power 130 (1). doi:10 .1115/ 1.2771240

[13] J. H. Griffin, An Integrated Approach for Friction Damper Design, Journal of Vibration and Acoustics 1 (April 1990) (1990) $175-181$.

[14] G. C. K. Yeh, Forced vibrations of a two degree of freedom system with combined coulomb and viscous damping, The Journal of the Acoustical Society of America 39 (1) (1966) 14-24. doi:doi.org/10.1121/1.1909863

[15] M. H. Jareland, A parametric study of a cottage-roof damper and comparison with experimental results, in: Proceedings of ASME TURBOEXPO, 2001, pp. 1-9.

[16] L. Panning, W. Sextro, K. Popp, Spatial Dynamics of Tuned and Mistuned Bladed Disks with Cylindrical and Wedge-Shaped Friction Dampers, International Journal of Rotating Machinery 9 (2003) 219-228. doi : 10.1155/S1023621X03000198

[17] E. Cigeroglu, N. An, C. H. Menq, Forced Response Prediction of Constrained and Unconstrained Structures Coupled Through Frictional Contacts, Journal of Engineering for Gas Turbines and Power 131 (2) (2008) 919. doi:10.1115/1.2940356

[18] C. M. Firrone, S. Zucca, M. M. Gola, The effect of underplatform dampers on the forced response of bladed disks by a coupled static/dynamic harmonic balance method, International Journal of Non-Linear Mechanics 46 (2) (2011) 363-375. doi:10.1016/j.ijnonlinmec. 2010.10 .001 
[19] S. Zucca, C. M. Firrone, M. M. Gola, Modeling Underplatform Dampers for Turbine Blades: a Refined Approach in the Frequency Domain, Journal of Vibration and Control 19 (7) (2013) 1087-1102. doi:10.1177/1077546312440809

[20] E. P. Petrov, D. J. Ewins, Advanced Modeling of Underplatform Friction Dampers for Analysis of Bladed Disk Vibration, Journal of Turbomachinery 129 (1) (2007) 143. doi:10.1115/1.2372775

[21] E. P. Petrov, D. J. Ewins, Analytical Formulation of Friction Interface Elements for Analysis of Nonlinear Multi-Harmonic Vibrations of Bladed Disks, Journal of Turbomachinery 125 (2) (2003) 364. doi:10.1115/1.1539868

[22] E. P. Petrov, Explicit Finite Element Models of Friction Dampers in Forced Response Analysis of Bladed Disks, Journal of Engineering for Gas Turbines and Power 130 (2) (2008) 022502. doi:10.1115/1.2772633

[23] M. Krack, L. P. von Scheidt, J. Wallaschek, A high-order harmonic balance method for systems with distinct states, Journal of Sound and Vibration 332 (21) (2013) 5476-5488. doi:doi:10.1016/j.jsv.2013.04.048

[24] L. Salles, L. Blanc, F. Thouverez, A. Gouskov, P. Jean, Dual time stepping algorithms with the high order harmonic balance method for contact interfaces with fretting-wear, Journal of Engineering for Gas Turbines and Power 134 (3) (2012) 032503 (1-7).

[25] A. Grolet, F. Thouverez, On a new harmonic selection technique for harmonic balance method, Mechanical Systems and Signal Processing 30 (2012) 43-60. doi:10.1016/j.ymssp.2012.01.024

[26] R. R. Craig, M. C. C. Bampton, Coupling of Substructures for Dynamic Analyses, AIAA Journal 6 (7) (1968) 1313-1319.

[27] B. Besselink, U. Tabak, A. Lutowska, N. van de Wouw, H. Nijmeijer, D. Rixen, M. Hochstenbach, W. Schilders, A comparison of model reduction techniques from structural dynamics, numerical mathematics and systems and control, Journal of Sound and Vibration 332 (19) (2013) $4403-4422$. doi:doi: $10.1016 / j . j s v .2013 .03 .025$

[28] E. P. Petrov, A High-Accuracy Model Reduction for Analysis of Nonlinear Vibrations in Structures With Contact Interfaces, Journal of Engineering for Gas Turbines and Power 133 (10) (2011) 102503. doi:10.1115/1.4002810

[29] J. S. Green, Controlling forced response of a high pressure turbine blade Ph.D. thesis, KTH Royal Institute of Technology (2006). URL http://www.diva-portal.org/smash/get/diva2:10533/FULLTEXT01.pdf

[30] F. Pfeiffer, M. Hajek, Stick-slip motion of turbine blade dampers, Phil. Trans. R.Soc. Lond. A 338 (1992) 503-517.

[31] K.-H. Koh, J. H. Griffin, Dynamic Behavior of Spherical Friction Dampers and Its Implication to Damper Contact Stiffness, Journal of Engineering for Gas Turbines and Power 129 (2) (2007) 511. doi:10.1115/1.2436547

[32] C. M. Firrone, Measurement of the kinematics of two underplatform dampers with different geometry and comparison with numerical simulation, Journal of Sound and Vibration 323 (1-2) (2009) 313-333. doi:10.1016/j.jsv.2008.12.019

[33] L. Pesaresi, L. Salles, R. Elliot, A. Jones, J. S. Green, C. W. Schwingshackl, Numerical and experimental investigation of an underplatform damper test rig, Applied Mechanics and Materials 849 (2016) 1-12. doi:10.4028/www.scientific.net/AMM. 849.1

[34] M. M. Gola, L. Tong, A direct experimentalnumerical method for investigations of a laboratory under-platform damper behavior, International Journal of Solids and Structures 51 (2526) (2014) 4245 - 4259. doi:http://dx.doi.org/10.1016/j.ijsolstr.2014.08. 011

[35] M. M. Gola, C. Gastaldi, Understanding Complexities in Underplatform Damper Mechanics, Proceedings of ASME TURBOEXPO (2014) V07AT34A002doi:doi:10.1115/GT2014-25240

[36] I. a. Sever, E. P. Petrov, D. J. Ewins, Experimental and Numerical Investigation of Rotating Bladed Disk Forced Response Using Underplatform Friction Dampers, Journal of Engineering for Gas Turbines and Power 130 (4) (2008) 042503. doi:10.1115/1.2903845

[37] T. Berruti, C. M. Firrone, M. M. Gola, A Test Rig for Noncontact Traveling Wave Excitation of a Bladed Disk With Underplatform Dampers, Journal of Engineering for Gas Turbines and Power 133 (3) (2011) 032502. doi:10.1115/1.4002100

[38] R. K. Giridhar, P. V. Ramaiah, G. Krishnaiah, S. G. Barad, Gas Turbine Blade Damper Optimization Methodology, Advances in Acoustics and Vibration 2012 (2012) 1-13. doi:10.1155/2012/316761

[39] S. Smith, J. Bilbao-Ludena, S. Catalfamo, M. Brake, P. Reu, C. W. Schwingshackl, The effects of boundary conditions, measurement techniques, and excitation type on measurements of the properties of mechanical joints, in: Proceedings of the Society for Experimental Mechanics IMAC Conference, 2016.

[40] C. W. Schwingshackl, C. Joannin, L. Pesaresi, J. S. Green, N. Hoffmann, Test method development for nonlinear damping extraction of dovetail joints, in: Proceedings of the Society for Experimental Mechanics IMAC Conference, 2014.

[41] V. Ruffini, C. Schwingshackl, J. Green, Ldv measurement of local nonlinear contact conditions of flange joint, in: Topics in Nonlinear Dynamics, Volume 1: Proceedings of the 31st IMAC, A Conference on Structural Dynamics, 2013, Vol. 35, Springer Science \& Business Media, 2013, p. 159.

[42] C. W. Schwingshackl, Measurement of Friction Contact Parameters for Nonlinear Dynamic Analysis, in: Proceedings of the Society for Experimental Mechanics IMAC Conference, 2012.

[43] S. Medina, D. Nowell, D. Dini, Analytical and numerical models for tangential stiffness of rough elastic contacts, Tribology Letters 49 (1) (2013) 103-115. doi:10.1007/s11249-012-0049-y

[44] C. Putignano, M. Ciavarella, J. Barber, Frictional energy dissipation in contact of nominally flat rough surfaces under harmonically varying loads, Journal of the Mechanics and Physics of Solids 59 (12) (2011) 2442 - 2454. doi:doi:10.1016/j.jmps.2011.09.005

[45] C. W. Schwingshackl, E. P. Petrov, D. J. Ewins, Effects of Contact Interface Parameters on Vibration of Turbine Bladed Disks With Underplatform Dampers, Journal of Engineering for Gas Turbines and Power 134 (3) (2012) 032507. doi:10.1115/1.4004721 